\title{
Isotopic Evidence for Long-Distance Connections of the AD Thirteenth-Century Promontory Caves Occupants
}

\author{
Jessica Z. Metcalfe (D, John W. Ives, Sabrina Shirazi, Kevin P. Gilmore, Jennifer Hallson, \\ Fiona Brock, Bonnie J. Clark, and Beth Shapiro
}

The Promontory caves (Utah) and Franktown Cave (Colorado) contain high-fidelity records of short-term occupations by groups with material culture connections to the Subarctic/Northern Plains. This research uses Promontory and Franktown bison dung, hair, hide, and bone collagen to establish local baseline carbon isotopic variability and identify leather from a distant source. The ankle wrap of one Promontory Cave 1 moccasin had a $\delta^{13} C$ value that indicates a substantial $C_{4}$ component to the animal's diet, unlike the $C_{3}$ diets inferred from 171 other Promontory and northern Utah bison samples. We draw on a unique combination of multitissue isotopic analysis, carbon isoscapes, ancient DNA (species and sex identification), tissue turnover rates, archaeological contexts, and bison ecology to show that the high $\delta^{13} \mathrm{C}$ value was not likely a result of local plant consumption, bison mobility, or trade. Instead, the bison hide was likely acquired via long-distance travel to/from an area of abundant $C_{4}$ grasses far to the south or east. Expansive landscape knowledge gained through long-distance associations would have allowed Promontory caves inhabitants to make well-informed decisions about directions and routes of movement for a territorial shift, which seems to have occurred in the late thirteenth century.

Keywords: stable isotopes, migration, mobility, bison, Great Basin, Promontory Caves, Franktown Cave, Dene, Athapaskan, Apachean

Las cuevas Promontory (Utah) y la Cueva Franktown (Colorado) contienen registros de alta fidelidad en cuanto a las ocupaciones de corto término de grupos humanos con cultura material conectada al Subártico/Planicies del Norte. Esta investigación utiliza excremento, pelo, piel de cuero y colágeno del hueso de bisonte provenientes de los sitios Promontory y Franktown para establecer una base de datos isotópicos locales y variabilidad isotópica de carbono e identifica la piel de cuero de procedencia lejana. La envoltura de tobillo de un mocasín recuperado de la Cueva 1 de Promontory tuvo un valor $\delta^{13} \mathrm{C} q u e$ indica un componente $C_{4}$ considerable en la dieta del animal, lo que es un resultado distinto a las dietas $C_{3}$ que se identificaron a partir del análisis de 171 muestras de bisonte provenientes de Promontory y el norte de Utah. Aplicamos una combinación única de análisis isotópico de tejidos múltiples, mapas isotópicos ("isoscapes") de carbono, ADN antiguo (identificación de sexo y especie), tasa de remodelación de tejidos, contextos arqueológicos y la ecología del bisonte para demonstrar que el valor alto $\delta^{13}$ C probablemente no es un resultado debido al consumo de plantas locales, movilidad del bisonte o comercio. En cambio, la piel de cuero del bisonte fue probablemente adquirida a través de viajes de larga distancia hacia zonas con

Jessica Z. Metcalfe (jmetcal1 @ lakeheadu.ca, corresponding author) — Department of Anthropology, Lakehead University, 955 Oliver Road, Thunder Bay, Ontario P7B 5E1, Canada

John W. Ives and Jennifer Hallson a Department of Anthropology, University of Alberta, Edmonton, Alberta T6G 2E3, Canada

Sabrina Shirazi 匹 Department of Ecology and Evolutionary Biology, University of California, Santa Cruz, 1156 High Street, Santa Cruz, CA 95064, USA

Kevin P. Gilmore $\square$ HDR, 9781 S. Meridian Boulevard, Suite 400, Englewood, CO 80112, USA

Fiona Brock $\square$ Cranfield Forensic Institute, Cranfield University, Defence Academy of the United Kingdom, Shrivenham SN6 8LA, UK

Bonnie J. Clark - Department of Anthropology, University of Denver, 2000 E. Asbury Avenue, Denver, CO 80208, USA Beth Shapiro $\mathbf{a}$ Department of Ecology and Evolutionary Biology and Howard Hughes Medical Institute, University of California, Santa Cruz, 1156 High Street, Santa Cruz, CA 95064, USA

American Antiquity 86(3), 2021, pp. 526-548

Copyright (C) The Author(s), 2021. Published by Cambridge University Press on behalf of the Society for American Archaeology. This is an Open Access article, distributed under the terms of the Creative Commons Attribution licence (http://creativecommons.org/licenses/by/4.0/), which permits unrestricted re-use, distribution, and reproduction in any medium, provided the original work is properly cited.

doi:10.1017/aaq.2020.116 
pastos $C_{4}$ abundantes muy al sur o al este. El conocimiento extenso sobre el paisaje obtenido a través de asociaciones de larga distancia debió permitir a los habitantes de las cuevas Promontory tomar decisiones bien informadas sobre las direcciones y rutas de movimiento para realizar un cambio territorial, el cual parece haber ocurrido a finales del sigo XIII.

Palabras clave: isótopos estables, migración, movilidad, bisonte, Gran Cuenca, Cuevas Promontory, Cueva Franktown, Dene, Atapascano, Apacheano

The standard practice of eliminating apparent "anomalous" results preclude[s] the possibility of discovering the unexpected, identifying the unusual, and uncovering individual shortlived visitation events [Seymour 2010:158].

In the quotation above, Seymour (2010) argues that chronometric dating outliers can provide critical evidence of periodic short-term occupations, particularly among highly mobile populations. In this article, we argue that Seymour's statement is even more relevant for stable isotope analyses. In radiocarbon dating, tiny amounts of contaminant carbon can have extremely large effects on measurement results and hence on inferred dates (e.g., Wood et al. 2016; Zazula et al. 2014). In contrast, stable carbon isotope $\left(\delta^{13} \mathrm{C}\right)$ values measured from purified materials such as bone collagen and hair keratin are less susceptible to contamination. Only large-scale biochemical alteration and/or contamination significantly alters $\delta^{13} \mathrm{C}$ values, and such changes can usually be detected using quality-control indicators (Ambrose 1990; van Klinken 1999). Consequently, a single carbon stable isotope outlier can provide robust insight into aspects of human or animal behavior, including migration and mobility. Outlying $\delta^{18} \mathrm{O}$ and ${ }^{87} \mathrm{Sr} /{ }^{86} \mathrm{Sr}$ values measured from tooth enamel are well established as indicators of human and animal migrations where local isotopic baselines are well constrained (e.g., Bentley and Knipper 2005; Hodell et al. 2004). In some contexts, outlying $\delta^{13} \mathrm{C}$ values obtained from animal tissues may provide similarly valuable insights (Hobson and Wassenaar 2019). Obtaining a robust baseline of local isotope values is crucial for this type of research.

While engaged in a comprehensive AMS radiocarbon dating study of early Promontory Phase materials from the Promontory caves of northern Utah (Steward's [1937] Promontory Culture), we noticed that one leather sample from the ankle wrap of a fragmentary moccasin recovered in our 2011 excavations had a $\delta^{13} \mathrm{C}$ value $(-12.5 \%$ o $)$ that was much higher than any of the other 51 values obtained from materials in the same archaeological context (Ives, Froese, et al. 2014). In contrast to the moccasin's unusual $\delta^{13} \mathrm{C}$ value, its AMS and calibrated calendar dates $(725 \pm 24$ years BP, OxA-25234; 95\% probability range AD 1252-1290) fell firmly within both the measured and Bayesian-modeled ranges of the other Promontory samples (Ives, Froese, et al. 2014).

In this study, we examine the significance of this isotopically unusual moccasin leather. Is it feasible that its high $\delta^{13} \mathrm{C}$ value came from a bison consuming plants near the Promontory caves? Or did it come from somewhere else? If so, where? To address these questions, we present a local carbon isotope baseline of 157 samples of Promontory caves bison dung, hair, hide, and bone collagen; 14 samples of modern Antelope Island bison hair and dung; and 8 samples of Promontory-era Franktown Cave bison hair and hide (Figure 1). We use these isotopic data along with a grassland carbon isoscape, ancient DNA (species and sex identification), tissue turnover rates, and analogies with modern bison behavior to evaluate competing explanations for the outlying Promontory mocca$\sin \delta^{13} \mathrm{C}$ value. These alternative hypotheses include the following: (1) the leather was from a local Promontory-area animal that consumed unusually large amounts of $\mathrm{C}_{4}$ and/or CAM plants, (2) the leather was from an animal that migrated into the Promontory region from a faraway location, (3) the leather was obtained from a faraway location through trade, or (4) the leather was obtained during long-distance travel by people.

\section{The Promontory Caves}

The Promontory caves were excavated in 19301931 by Julian Steward (1937) and again between 2011 and 2014 by Ives, Janetski, and 


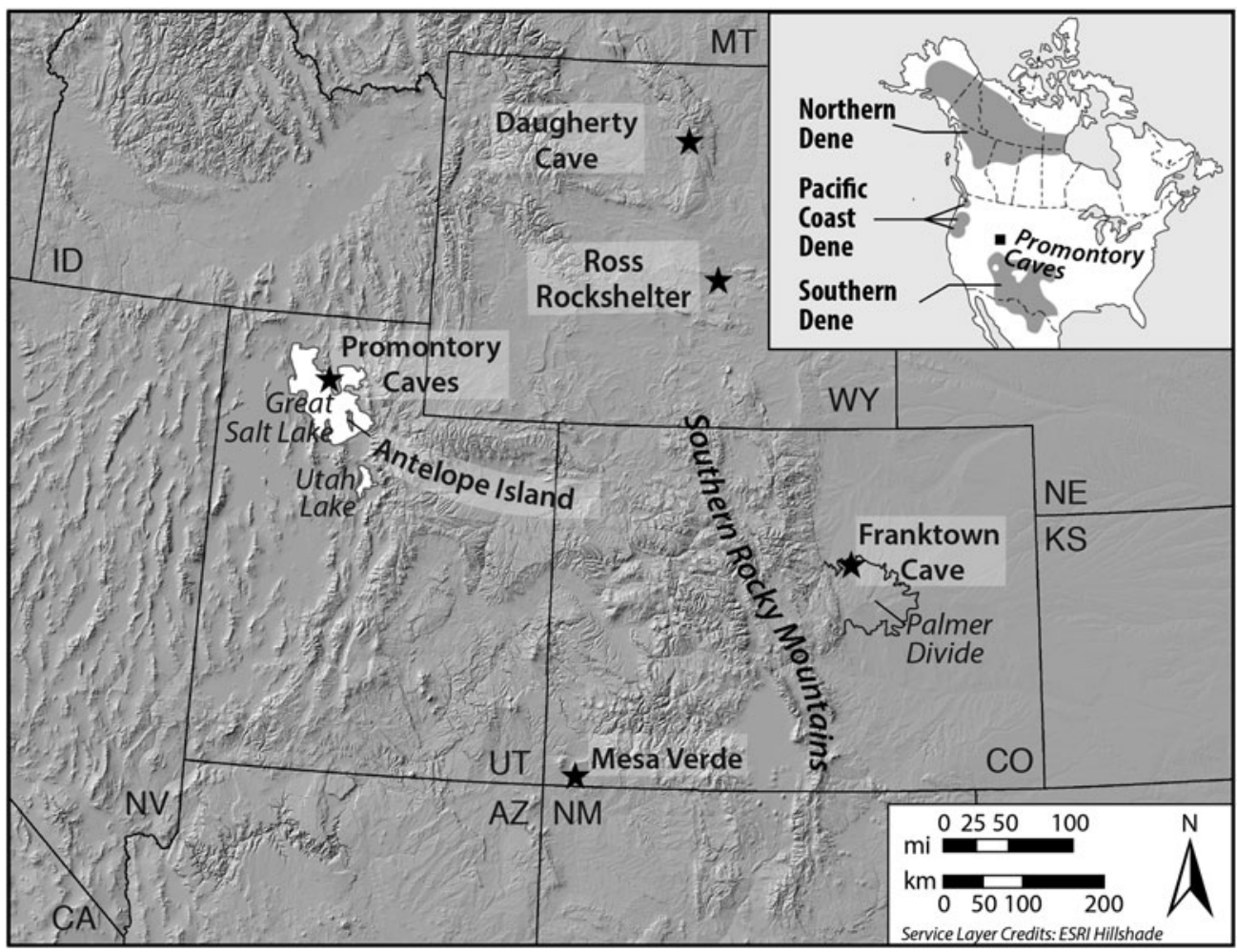

Figure 1. Locations mentioned in the text, including sampling locations (Promontory Caves, Franktown Cave, Antelope Island) and other locations with Promontory-style moccasins (Daugherty Cave, Ross Rockshelter, Mesa Verde). Inset map shows colonial-era locations of Dene groups in North America.

colleagues (Billinger and Ives 2015; Ives 2014, 2020; Ives, Froese, et al. 2014). Both research episodes recovered an abundance of exquisitely preserved organic remains, including moccasins, basketry, and cordage; many varied gaming pieces; a complete range of stone and bone tools, from hunting weaponry to hide processing tools; ceramics; items of adornment; indications of ceremonial life such as rock art and incised tablets; and animal bone, hide, hair, and dung (Figure 2; Ives, Froese, et al. 2014; Thomas 2018; Yanicki and Ives 2017). Two aspects of the Promontory occupation are abundantly clear: bison and other large game animals were intensively hunted near the caves, and Cave 1 (42BO1) was a residential location at which women, men, subadults, and children were present at various seasons for extended periods of time over one or two human generations.

Cave 1 contains faint, discontinuous traces of Archaic use, but the vast majority of deposits are
Promontory Culture. Cave 2 (42BO2) has some preceding Archaic occupations and a substantial Promontory Culture layer, although fewer deposits than Cave 1. Steward (1937) found that the Promontory Culture layer in Cave 1 covered much of the roughly $240 \mathrm{~m}^{2}$ of habitable space and averaged about $65 \mathrm{~cm}$ in depth. Although we made similar stratigraphic findings in Steward's Trench B area, in his Trench A area toward the front of Cave 1, we discovered intact midden deposits that were $2 \mathrm{~m}$ deep. These Cave 1 deposits produced a large assemblage of radiocarbon dates with a tight range of ages Bayesian modeled between AD 1240 and 1295 (95\% probability estimate; Ives, Froese, et al. 2014).

Although our excavations were minor (roughly $3 \mathrm{~m}^{3}$ of deposit) we recovered more than 30,000 whole and fragmentary faunal remains, supporting Steward's (1937) contention that the Promontory cave occupants focused almost exclusively on large-game hunting, particularly 


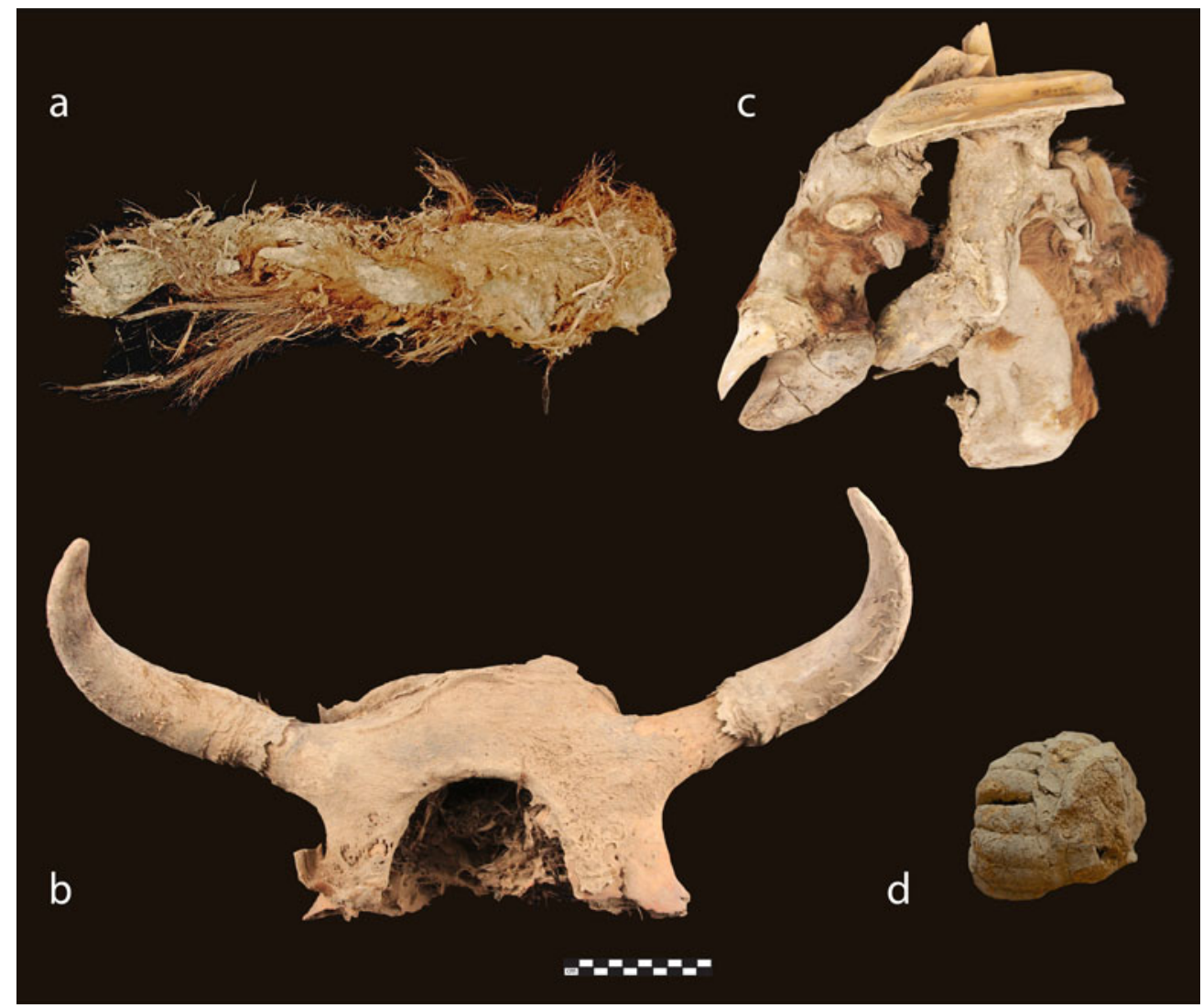

Figure 2. Exceptionally preserved late thirteenth-century bison materials from Promontory Cave 1 (42BO1), including (a) hide and long hair (FS-203); (b) skull with hide and horn sheaths (FS-1255); (c) limb with hair, hide, hoof, and bone (FS-1); and (d) dung (FS-703). Photographs by by J.W. Ives (a, b, c) and J. Hallson (d).

of bison and pronghorn (see Johansson 2013). Our excavations yielded few small mammals or aquatic resources and scant evidence of seed processing (Ives, Johansson, et al. 2014). The Promontory Culture occupation postdates the cessation of maize horticulture among the local Fremont, although relatively sedentary populations were still present in the area, including the nearby Chournos Springs site (e.g., Yanicki 2019).

For Promontory Cave 1, discard and accumulation formulae along with space syntax analyses suggest a group size of roughly 35 persons, an estimate of 1.8 million whole and fragmentary faunal remains, and an extrapolated total of 2,400 moccasins within the deposits (Hallson 2017; Lakevold 2017). Seasonality indicators are still being refined, but a range of evidence - from deer skulls with cast antlers to abundant bulrush seeds (by-products of summer mat making)_-indicates that Cave 1 was occupied during various seasons of the year. Obsidian sourcing and ceramic evidence further suggest that Promontory cave occupants ranged into southern Idaho (Arkush 2016; Ives 2020). Altogether, the Cave 1 Promontory Culture deposits reflect a sudden, substantial, but seasonally intermittent residential occupation of about 55 years' duration, supported by intensive large-game hunting. The high volume of material culture resulted from the domestic presence of many children, subadults, and adult women and men.

Steward (1937) concluded that the Promontory Culture deposits had been created by a population with northern Plains and Subarctic affinities. He voiced the suspicion that they were Dene ancestors who had lingered long enough to absorb material culture characteristics 
from neighboring terminal Fremont communities. The potential attribution of a protoApachean identity to the Promontory cave occupants is an underlying interest in our research group. Triggered by the massive White River Ash east lobe eruption at AD 846-848, a small founding population of Apachean ancestors may have gradually made their way south in ensuing centuries (e.g., Achilli et al. 2013; Kristensen, Andrews, et al. 2019; Kristensen, Hare, et al. 2019; Kristensen et al. 2020). Promontory caves material culture linkages to the Subarctic include moccasins constructed in a characteristically Subarctic style (Bata Shoe Museum classifications BSM 2[Bb] and BSM 2[Ab]), fine leather from a sophisticated hide-processing tradition, and distinctive artifacts such as chi-thos (tabular bifaces used for hide softening), which are common in the Subarctic but unknown in Fremont assemblages (Ives, Froese, et al. 2014; Webber 1989). The Promontory moccasins stand in visible contrast to many surrounding forms of footwear, from hide and plant fiber sandals to hock moccasins (e.g., Clark 1885:257; Hatt 1916; Lycett 2015). The Promontory moccasins are completely unlike Fremont footwear or most other footwear in a broader arc in interior western North America (Ives 2020:103-104; Ives, Froese, et al. 2014: 626-627). Like many other forms of clothing, moccasins signal cultural identity (in the caliber of leather, the knowledge of requisite patterns, execution of fine motor skills, and decorative techniques). As for absorbing regional traits, the rare basketry in the Promontory caves is characteristic of Great Basin forms, and Promontory pottery was influenced by Fremont forms (Ives, Froes, et al. 2014; Yanicki 2019). We are nevertheless mindful of other explanations for the Promontory Culture expressions. These would include the possibility of Kiowa involvement (although this would have its own Dene dimension, given the long-term Kiowa relationship with Ndee [Plains Apache] peoples) or the prospect that Promontory expressions might be yet more broadly polyethnic in character (Ives 2014, 2020; Mooney 1898; Ortman and McNeil 2017).

Whatever the cultural identities involved, the Promontory record raises an important and broader set of issues. The AD thirteenth century in western North America was turbulent, featuring profound social and environmental shifts in which hunter-gatherer mobility imparted significant advantages for some groups as more sedentary societies vanished (as with Fremont) or retracted (as with Puebloan communities; e.g., Benson et al. 2007; Kohler et al. 2014; Robinson et al. 2021). By the time of Spanish contact, there existed a variety of Plains-Puebloan interactions founded on the trade of Plains bison and other products for Puebloan goods, and a spectrum of relationships ranging from enclavement among Puebloan communities (as with the Jicarilla Apache) to raiding (Eiselt 2012). Irrespective of the precise cultural identity of the people who produced it, the Promontory record suggests that the foundation for later relationships between Plains bison-oriented economies and more sedentary villagers was being laid in the thirteenth century.

\section{Franktown Cave}

Franktown Cave (5DA272), Colorado (south of Denver on the Palmer Divide; Figure 1), has material cultural affiliations with the late thirteenth-century Promontory caves. Like Promontory, Franktown Cave's assemblage includes Promontory-style ceramic sherds, a netted gaming hoop, incised wood (probably gaming pieces), sinew-backed bows, and two moccasins of the BSM-2(Bb) type (Gilmore 2005). According to Bayesian modeling (95\% probability estimates), these Franktown artifacts date to AD 1180 1280, which overlaps and slightly predates the occupation of the Promontory caves themselves (Gilmore et al. 2021; Gilmore, Hamilton, and Ives 2017). Both Franktown Cave and Promontory Cave 1 occupy easily defensible positions overlooking the surrounding area, which may reflect only partial integration with local groups in a dynamically changing thirteenth-century sociopolitical landscape (Lakevold 2017). The Late Prehistoric occupants of Franktown Cave and the Promontory caves shared a distinctive material culture heritage (Ives, Froese, et al. 2014).

\section{Carbon Isotopes in Bison Diets: Vegetation}

Bison are herbivores and therefore derive their carbon isotope compositions from plants. 
Although they are primarily grazers, bison also occasionally consume sedges, forbs, and, less frequently, woody plants (Gogan et al. 2010). Bison prefer nutritious new growth, particularly $\mathrm{C}_{3}$ grasses during cooler seasons and $\mathrm{C}_{4}$ grasses during summer (Gogan et al. 2010). Across the Great Plains, modern free-ranging bison tissue $\delta^{13} \mathrm{C}$ values directly correlate with local abundances of $\mathrm{C}_{3}$ and $\mathrm{C}_{4}$ grasses (Hoppe et al. 2006).

Carbon isotope analysis can be used to distinguish between plants using different photosynthetic pathways: $\mathrm{C}_{3}$ (cool-season grasses, forbs, and most woody vegetation), $\mathrm{C}_{4}$ (warm-season grasses and some shrubs), and CAM (cacti and succulents). Today, northern Utah, southern Idaho, and western Wyoming vegetation is dominated by shrubs and $\mathrm{C}_{3}$ grasses. Although substantial $\mathrm{C}_{3}$ grasslands exist in this region, $\mathrm{C}_{4}$ grasses are relatively rare (Cotton et al. 2016). Microhabitats that support higher abundances of $\mathrm{C}_{4}$ grasses (e.g., Distichlis spicata, Sporobolus airoides), however, do exist (Morden et al. 1986). $\mathrm{C}_{4}$ shrubs (e.g., Atriplex spp.) and CAM cacti and succulents (e.g., Opuntia spp.) are relatively abundant in northern Utah (Coltrain and Leavitt 2002; Sandquist and Ehleringer 1995; Ugan and Coltrain 2012), but are not known to be components of bison diets.

Modern $\mathrm{C}_{3}$ plants have a mean $\delta^{13} \mathrm{C}$ around $-27 \%$, with a range from about $-37 \%$ o to $-20 \%$ o (Kohn 2010). Modern plant values are lower than those of plants that grew before the onset of large-scale human industrial activities, which are continually lowering the $\delta^{13} \mathrm{C}$ of atmospheric $\mathrm{CO}_{2}$ (e.g., Graven et al. 2017). The Holocene preindustrial $\delta^{13} \mathrm{C}$ of $\mathrm{CO}_{2}$ was about $-6.4 \%$ (Schmitt et al. 2012), which is $1.6 \%$ o higher than the postindustrial atmospheric $\mathrm{CO}_{2}$ $\delta^{13} \mathrm{C}$ of $-8.0 \%$ used by Kohn (2010) to standardize his modern plant values. Thus, preindustrial $\mathrm{C}_{3}$ plants should have had a global mean $\delta^{13} \mathrm{C}$ of around $-25.4 \%$ and a range between about -35.4 and $-18.4 \%$ o. In a global survey of modern $\mathrm{C}_{3}$ plants, only plants from hyper-arid locations (mean annual precipitation $<10 \mathrm{~mm} /$ year) and genus Pinus had $\delta^{13} \mathrm{C}$ values $>-23 \%$ (i.e., preindustrial plant $\delta^{13} \mathrm{C}>-21.4 \%$; Kohn 2010). Because bison do not consume pine, preindustrial bison with inferred dietary $\delta^{13} \mathrm{C}$ significantly higher than $-21.4 \%$ can reasonably be inferred to indicate consumption of $\mathrm{C}_{4}$ or CAM plants. Given bison dietary preferences, such values would most likely indicate consumption of $\mathrm{C}_{4}$ grasses.

There is a very clear separation between the $\delta^{13} \mathrm{C}$ values of $\mathrm{C}_{3}$ and $\mathrm{C}_{4} / \mathrm{CAM}$ plants: their distributions are nonoverlapping and their means differ by about 14\%o (Cerling et al. 1999; O'Leary 1988). Modern $\mathrm{C}_{4}$ plant $\delta^{13} \mathrm{C}$ values typically fall within the range of $-16 \%$ to $-10 \%$ o, and modern CAM plants that are actively utilizing the CAM photosynthetic pathway also tend to have $\delta^{13} \mathrm{C}$ values within this range (O'Leary 1988; Sternberg et al. 1984). Modern $\mathrm{C}_{4}$ plants from Utah fall within this range as well (Coltrain and Leavitt 2002). Accounting for a Suess Effect shift of 1.6\%o, the preindustrial $\mathrm{C}_{4} / \mathrm{CAM}$ plant range would have been about $-14.4 \%$ o to $-8.4 \%$. A recent study of wellpreserved Late Pleistocene and Early Holocene plants from Utah's Colorado Plateau resulted in CAM plant (Opuntia sp.) $\delta^{13} \mathrm{C}$ values between $-10.8 \%$ and $-9.8 \%$ o (mean $=-10.3 \%$ ), in agreement with these global generalizations (Metcalfe and Mead 2019).

\section{Carbon Isotopes in Bison Tissues: Diet Estimates}

To directly compare animal tissues to one another or to plant $\delta^{13} \mathrm{C}$ values, one must account for isotopic fractionations that occur when plant carbon is incorporated into each type of biological tissue. Large herbivore bone collagen $\delta^{13} \mathrm{C}$ values are about 5\%o higher than the $\delta^{13} \mathrm{C}$ of plants in their diet (Drucker et al. 2008; Tieszen 1994). Hair $\delta^{13} \mathrm{C}$ values are on average about $3 \%$ higher than $\delta^{13} \mathrm{C}$ of plants in the diet (Bocherens et al. 2014; Crowley et al. 2010; Drucker et al. 2008). Dung $\delta^{13} \mathrm{C}$ values are typically $1 \%$ o lower than those of diet (Sponheimer et al. 2003). The isotopic compositions of skin (hide) have seldom been studied, but Tieszen (1994) showed that for one bison, skin/hide $\delta^{13} \mathrm{C}$ was about $0.5 \%$ lower than bone collagen and about $2.0 \%$ higher than hair, which is broadly consistent with experimental studies of manatee and seal skin (Alves-Stanley and Worthy 2009; Ames et al. 1996; Hobson et al. 1996). 
Tissues form over different time scales and can therefore provide information about animal diets over different durations of time. Bison dung represents the diet consumed 1-3 days immediately prior to deposition (Rutley and Hudson 2000; Schaefer et al. 1978), and therefore likely includes vegetation consumed in the geographical region of deposition. In contrast, bone collagen $\delta^{13} \mathrm{C}$ values are derived from the diet consumed over a period of years (Hedges et al. 2007; Hobson and Clark 1992). A recent study found that the cortical bone of bear, deer, serow, and macaque formed primarily during adolescence (i.e., the active bone-growth phase) rather than throughout the animal's life (Matsubayashi and Tayasu 2019). Thus, in some instances, bison cortical bone collagen may represent the diet several years prior to death. If an old animal migrated into a new area during its adolescence, its bone collagen could represent foods consumed in locations quite distant from where the bones were recovered. If animals did not migrate, bone collagen would provide a record of local diet. Regardless, bone collagen integrates diet over a long period of time. Hair contains a short- or medium-term record of diet, depending on the sampling strategy and the hair's growth rate. When hair is sampled in segments, it is possible to recover a time series of changes over a period of days, weeks, or months. Skin is constantly re-forming and therefore represents diet relatively close to the time of death. Skin turnover rates have not been studied in terrestrial mammals. Manatee and dolphin carbon isotope "half-lives" (time to reach half-equilibrium with new values after a controlled-diet switch) ranged from about two weeks to four months, so time to reach full equilibrium would be on the order of several months or more (Alves-Stanley and Worthy 2009; Browning et al. 2014). Based on these turnover rates, bone and skin $\delta^{13} \mathrm{C}$ values would be less likely to record brief and/or occasional consumption of isotopically distinct plants, whereas dung and hair $\delta^{13} \mathrm{C}$ would hold greater potential for recording such brief deviations from a typical diet.

Based on the research described above, we convert bison dung, hair, hide, or bone collagen $\delta^{13} \mathrm{C}$ to short-, medium-, and long-term diet $\delta^{13} \mathrm{C}$ according to the following equations:
Short-term bison diet 1-3 days prior to deposition:

$$
\delta^{13} \mathrm{C}_{\text {diet }}=\delta^{13} \mathrm{C}_{\text {dung }}+1
$$

Medium-/short-term bison diet (weeks/months):

$$
\delta^{13} C_{\text {diet }}=\delta^{13} C_{\text {hair }}-3
$$

Medium-term bison diet (months):

$$
\delta^{13} \mathrm{C}_{\text {diet }}=\delta^{13} \mathrm{C}_{\text {hide }}-4.5
$$

Long-term diet (years):

$$
\delta^{13} C_{\text {diet }}=\delta^{13} C_{\text {bonecollagen }}-5
$$

A further conversion is needed to make diet $\delta^{13} \mathrm{C}$ values obtained from modern samples directly comparable to those from preindustrial archaeological samples. In this study, the $\delta^{13} \mathrm{C}$ values of modern Antelope Island samples collected in 2017 are converted to preindustrial equivalents by adding $+2.1 \%$, which accounts for the higher atmospheric $\mathrm{CO}_{2}$ in 2017 (when the samples were collected; ca. $-8.5 \%$ o) relative to the preindustrial global atmospheric $\mathrm{CO}_{2} \delta^{13} \mathrm{C}$ value of $-6.4 \%$ (Graven et al. 2017; Schmitt et al. 2012).

\section{Materials and Methods}

Bison samples (dung, hair, hide, and bone collagen) used for isotopic analysis are listed in Table 1. Detailed preparation and analytical methods are provided in the Supplemental Methods and explained here in brief. Hair and hide were cleaned and bone collagen was extracted following established procedures. Carbon isotope compositions $\left(\delta^{13} \mathrm{C}\right)$ and carbon and nitrogen contents $(\% \mathrm{C}, \% \mathrm{~N})$ were measured using an Elementar Vario Micro Cube elemental analyzer, coupled with an Isoprime stable isotope ratio mass spectrometer in continuousflow mode. Elemental compositions were calibrated using USGS-40. $\delta^{13} \mathrm{C}$ values were calibrated to the VPDB scale using USGS-40 and USGS-41 or 41a (accepted values $-26.39 \%$, $+37.63 \%$ o, and $+36.55 \%$, respectively). Internal check standards (gelatin, methionine, red lentil, amaranth; accepted values $-15.30 \%$ o, $-28.60 \%$, $-26.12 \%$, $-13.59 \%$, respectively) and sample 
Table 1. Isotopic and Elemental Data for Bison Tissues from Promontory (42BO1, 42BO2), Franktown (5DA272), and Antelope Island (AI).

\begin{tabular}{|c|c|c|c|c|c|c|c|c|}
\hline Material & Site & Sample & Element/Type & $\delta^{13} \mathrm{C}_{\text {tissue }}$ & $\delta^{13} \mathrm{C}_{\text {diet }}$ & $\% \mathrm{C}$ & $\% \mathrm{~N}$ & $\mathrm{C} / \mathrm{N}$ \\
\hline \multicolumn{9}{|c|}{ Bone collagen } \\
\hline & 42BO1 & FS-1 & Long bone & -19.2 & -24.2 & 38.5 & 14.0 & 3.2 \\
\hline & 42BO1 & FS-187.82 & Scapula & -20.0 & -25.0 & 41.6 & 14.3 & 3.4 \\
\hline & $42 \mathrm{BO} 2$ & FS-19 \#1 & Long bone & -19.8 & -24.8 & 41.0 & 14.8 & 3.2 \\
\hline & 42BO1 & FS-237.1 & Radius & -19.9 & -24.9 & 41.0 & 14.8 & 3.2 \\
\hline & 42BO1 & FS-237.24 & Tibia (left) & -17.9 & -22.9 & 40.6 & 14.7 & 3.2 \\
\hline & 42BO1 & FS-237.3 & Femur & -20.1 & -25.1 & 42.6 & 14.8 & 3.4 \\
\hline & 42BO1 & FS-575.38 & Long bone & -19.7 & -24.7 & 41.6 & 14.3 & 3.4 \\
\hline & 42BO1 & FS-575.39 & Femur & -19.4 & -24.4 & 42.4 & 15.1 & 3.3 \\
\hline & $42 \mathrm{BO} 2$ & FS-614.17 & Femur (right) & -18.7 & -23.7 & 40.6 & 14.8 & 3.2 \\
\hline & 42BO1 & FS-623.6 & Humerus (right) & -18.4 & -23.4 & 40.4 & 14.8 & 3.2 \\
\hline & $42 \mathrm{BO} 2$ & FS-702 & Rib & -19.6 & -24.6 & 41.8 & 15.2 & 3.2 \\
\hline & 42BO1 & FS-750.15 & cf Humerus & -19.8 & -24.8 & 43.0 & 15.2 & 3.3 \\
\hline \multicolumn{9}{|l|}{ Hair } \\
\hline & 42BO1 & FS-1 Ives \#1-1,3,5 & Coarse & -21.8 & -24.8 & 41.8 & 13.8 & 3.5 \\
\hline & 42BO1 & FS-1 Ives \#4 & Coarse & -22.0 & -25.0 & 42.0 & 14.1 & 3.5 \\
\hline & 42BO1 & FS-106-1 & Coarse & -21.7 & -24.7 & 41.6 & 13.8 & 3.5 \\
\hline & 42BO1 & FS-1169 & Coarse & -20.6 & -23.6 & 41.4 & 13.8 & 3.5 \\
\hline & 42BO1 & FS-1199 & Coarse & -20.4 & -23.4 & 41.0 & 13.6 & 3.5 \\
\hline & 42BO1 & FS-1305 & Coarse & -20.8 & -23.8 & 40.8 & 13.9 & 3.4 \\
\hline & 42BO1 & FS-1623- $3^{a}$ & Coarse & -21.8 & -24.8 & 45.5 & 14.8 & 3.6 \\
\hline & 42BO1 & FS-1623-4 $4^{\mathrm{a}}$ & Coarse & -22.0 & -25.0 & 42.7 & 13.7 & 3.6 \\
\hline & 42BO1 & FS-1623-5 $5^{\mathrm{a}}$ & Coarse & -21.9 & -24.9 & 44.2 & 14.5 & 3.6 \\
\hline & 42BO1 & FS-190-2 & Coarse & -20.0 & -23.0 & 42.1 & 14.1 & 3.5 \\
\hline & 42BO1 & FS-203 & Coarse & -22.0 & -25.0 & 42.1 & 13.5 & 3.6 \\
\hline & 42BO1 & FS-203 & Coarse & -21.1 & -24.1 & 41.4 & 13.8 & 3.5 \\
\hline & 42BO1 & FS-249-2 & Coarse & -20.3 & -23.3 & 41.6 & 13.5 & 3.6 \\
\hline & 42BO1 & FS-292 & Coarse and fine & -21.3 & -24.3 & 42.6 & 13.8 & 3.6 \\
\hline & 42BO1 & FS-311-1 & Coarse & -21.1 & -24.1 & 42.3 & 14.0 & 3.5 \\
\hline & $42 \mathrm{BO} 2$ & FS-38 & Fine & -22.1 & -25.1 & 43.5 & 14.2 & 3.6 \\
\hline & 42BO1 & FS-380 & Coarse & -21.1 & -24.1 & 46.0 & 15.0 & 3.6 \\
\hline & 42BO1 & FS- $404^{\mathrm{a}}$ & Coarse & -21.8 & -24.8 & 42.7 & 13.3 & 3.7 \\
\hline & 42BO1 & FS-417 & Fine & -21.7 & -24.7 & 44.1 & 14.4 & 3.6 \\
\hline & 42BO1 & FS-417 & Fine & -21.6 & -24.6 & 43.8 & 14.5 & 3.5 \\
\hline & 42BO1 & FS-607 & Fine & -21.3 & -24.3 & 44.0 & 15.0 & 3.4 \\
\hline & 42BO1 & FS-61-1 ${ }^{\mathrm{a}}$ & Coarse & -21.7 & -24.7 & 42.8 & 14.0 & 3.6 \\
\hline & 42BO1 & FS-61-2 ${ }^{\mathrm{a}}$ & Coarse & -21.6 & -24.6 & 44.6 & 15.3 & 3.4 \\
\hline & 42BO1 & FS-61-4 ${ }^{\mathrm{a}}$ & Coarse & -21.7 & -24.7 & 44.5 & 14.5 & 3.6 \\
\hline & 42BO1 & FS-656- $1^{\mathrm{a}}$ & Coarse & -21.2 & -24.2 & 42.9 & 13.8 & 3.6 \\
\hline & 42BO1 & FS-75-2 & Coarse & -22.2 & -25.2 & 41.1 & 13.4 & 3.6 \\
\hline & 42BO1 & FS-753-1 ${ }^{\mathrm{a}}$ & Coarse & -21.9 & -24.9 & 43.2 & 13.8 & 3.6 \\
\hline & 42BO1 & FS-753-2 ${ }^{\mathrm{a}}$ & Coarse & -21.7 & -24.7 & 44.6 & 14.0 & 3.7 \\
\hline & 42BO1 & FS-775-2 & Coarse & -21.7 & -24.7 & 42.8 & 14.4 & 3.5 \\
\hline & 42BO1 & FS-796 & Coarse & -21.0 & -24.0 & 45.8 & 14.9 & 3.6 \\
\hline & 42BO1 & FS-796 & Fine & -21.1 & -24.1 & 44.9 & 15.0 & 3.5 \\
\hline & 42BO1 & FS-853-1 & Coarse & -21.6 & -24.6 & 42.4 & 14.2 & 3.5 \\
\hline & 42BO1 & FS-878- $1^{\mathrm{a}}$ & Coarse & -21.6 & -24.6 & 44.0 & 14.7 & 3.5 \\
\hline & $\mathrm{AI}^{\mathrm{c}}$ & AI-u & Fine & -23.3 & -24.2 & 42.7 & 14.0 & 3.6 \\
\hline & $\mathrm{AI}^{\mathrm{c}}$ & AI-g & Coarse & -23.3 & -24.2 & 43.3 & 14.2 & 3.6 \\
\hline & $\mathrm{AI}^{\mathrm{c}}$ & AN-472 & Coarse & -23.3 & -24.2 & 44.1 & 14.6 & 3.5 \\
\hline & $\mathrm{AI}^{\mathrm{c}}$ & AN-473 & Fine & -23.4 & -24.3 & 43.1 & 14.4 & 3.5 \\
\hline & $\mathrm{AI}^{\mathrm{c}}$ & AN-475 & Coarse & -23.4 & -24.3 & 43.0 & 13.9 & 3.6 \\
\hline & $\mathrm{AI}^{\mathrm{c}}$ & AN-476a & Fine & -23.4 & -24.3 & 44.2 & 14.6 & 3.5 \\
\hline & $\mathrm{AI}^{\mathrm{c}}$ & AN-476 b & Fine & -23.3 & -24.2 & 44.4 & 14.6 & 3.5 \\
\hline
\end{tabular}


Table 1. Continued.

\begin{tabular}{|c|c|c|c|c|c|c|c|c|}
\hline Material & Site & Sample & Element/Type & $\delta^{13} \mathrm{C}_{\text {tissue }}$ & $\delta^{13} \mathrm{C}_{\text {diet }}$ & $\% \mathrm{C}$ & $\% \mathrm{~N}$ & $\mathrm{C} / \mathrm{N}$ \\
\hline & $\mathrm{AI}^{\mathrm{c}}$ & AN-474-1 b & Coarse & -23.1 & -24.0 & 46.1 & 13.8 & 3.9 \\
\hline & $\mathrm{AI}^{\mathrm{c}}$ & TOM-455 F2 \& F1 & Coarse & -12.6 & -15.6 & 43.4 & 13.8 & 3.7 \\
\hline & $\mathrm{AI}^{\mathrm{c}}$ & TOM-455 F3 & Coarse & -12.3 & -15.3 & 52.3 & 16.5 & 3.7 \\
\hline \multicolumn{9}{|c|}{ Hide/Leather } \\
\hline & $42 \mathrm{BO} 1$ & FS-305 ankle wrap & Moccasin ankle wrap & -12.9 & -17.4 & 34.9 & 12.4 & 3.3 \\
\hline & $42 \mathrm{BO} 1$ & FS-305 body & Moccasin body & -20.0 & -24.5 & 33.1 & 12.0 & 3.2 \\
\hline & $42 \mathrm{BO} 1$ & FS-1 Ives \#1 & Attached to limb & -19.5 & -24.0 & 37.6 & 13.4 & 3.3 \\
\hline & $42 \mathrm{BO} 1$ & FS-1 Ives \#4 & Attached to limb & -19.2 & -23.7 & 37.4 & 13.3 & 3.3 \\
\hline & 42BO1 & FS-1117 & Moccasin & -19.0 & -23.5 & 30.2 & 10.6 & 3.3 \\
\hline & $42 \mathrm{BO} 1$ & FS-1145 & Moccasin & -19.6 & -24.1 & 38.1 & 13.8 & 3.2 \\
\hline & $42 \mathrm{BO} 1$ & FS-1290 & Moccasin & -19.5 & -24.0 & 39.7 & 14.6 & 3.2 \\
\hline & $42 \mathrm{BO} 1$ & FS-1318 & Moccasin & -20.3 & -24.8 & 28.6 & 10.1 & 3.3 \\
\hline & $42 \mathrm{BO} 1$ & FS-1431 & Moccasin & -20.1 & -24.6 & 37.8 & 13.4 & 3.3 \\
\hline & $42 \mathrm{BO} 1$ & FS-1568 & Moccasin & -19.7 & -24.2 & 30.8 & 10.9 & 3.3 \\
\hline & $42 \mathrm{BO} 1$ & FS-1596 & Moccasin & -19.7 & -24.2 & 30.5 & 10.4 & 3.4 \\
\hline & $42 \mathrm{BO} 1$ & FS-1675 & Robe fragment & -19.7 & -24.2 & 40.4 & 14.8 & 3.2 \\
\hline & $42 \mathrm{BO} 1$ & FS-1676 & Robe fragment & -18.4 & -22.9 & 39.2 & 14.1 & 3.2 \\
\hline & $42 \mathrm{BO} 1$ & FS-1677 & Moccasin & -19.8 & -24.3 & 25.9 & 8.9 & 3.4 \\
\hline & $42 \mathrm{BO} 1$ & FS-203 & Strip, hair removed & -19.9 & -24.4 & 38.7 & 14.2 & 3.2 \\
\hline & $42 \mathrm{BO} 1$ & FS-292 & Strip, hair removed & -19.8 & -24.3 & 39.3 & 14.2 & 3.2 \\
\hline & $42 \mathrm{BO} 1$ & FS-61-4 & Removed from hair & -20.4 & -24.9 & 37.9 & 13.0 & 3.4 \\
\hline & $42 \mathrm{BO} 1$ & FS-775 & Removed from hair & -19.9 & -24.4 & 40.6 & 14.3 & 3.3 \\
\hline & $42 \mathrm{BO} 1$ & FS-945 & Moccasin & -20.2 & -24.7 & 24.1 & 8.0 & 3.5 \\
\hline & $42 \mathrm{BO} 1$ & OxA-18156 ${ }^{\mathrm{b}}$ & Moccasin sole & -20.4 & & & & \\
\hline & $42 \mathrm{BO} 1$ & OxA-18160 & Moccasin & -20.1 & & & & \\
\hline & $42 \mathrm{BO} 1$ & OxA-23853 & Moccasin & -20.2 & & & & \\
\hline & $42 \mathrm{BO} 1$ & OxA-23854 ${ }^{\mathrm{b}}$ & Moccasin & -20.3 & & & & \\
\hline & $42 \mathrm{BO} 1$ & OxA-23855 & Moccasin & -22.5 & & & & \\
\hline & $42 \mathrm{BO} 1$ & OxA-23856 & Moccasin & -19.5 & & & & \\
\hline & $42 \mathrm{BO} 1$ & OxA-23857 & Moccasin & -19.9 & & & & \\
\hline & $42 \mathrm{BO} 1$ & OxA-23886 & Moccasin & -20.0 & & & & \\
\hline & $42 \mathrm{BO} 1$ & OxA-23887 & Moccasin & -20.5 & & & & \\
\hline & $42 \mathrm{BO} 1$ & OxA-23888 & Moccasin & -18.7 & & & & \\
\hline & $42 \mathrm{BO} 1$ & OxA-23889 & Moccasin & -20.3 & & & & \\
\hline & $42 \mathrm{BO} 1$ & OxA-23890 & Moccasin & -20.2 & & & & \\
\hline & $42 \mathrm{BO} 1$ & OxA-23917 & Moccasin & -19.3 & & & & \\
\hline & $42 \mathrm{BO} 1$ & OxA-23918 & Moccasin & -20.4 & & & & \\
\hline & $42 \mathrm{BO} 1$ & OxA-23919 & Moccasin & -20.0 & & & & \\
\hline & $42 \mathrm{BO} 1$ & OxA-23920 & Moccasin & -18.7 & & & & \\
\hline & $42 \mathrm{BO} 1$ & OxA-23921 & Moccasin & -20.4 & & & & \\
\hline & $42 \mathrm{BO} 1$ & OxA-24002 & Moccasin & -19.9 & & & & \\
\hline & $42 \mathrm{BO} 1$ & OxA-24003 & Moccasin & -20.0 & & & & \\
\hline & $42 \mathrm{BO} 1$ & OxA-24004 & Moccasin & -19.8 & & & & \\
\hline & $42 \mathrm{BO} 1$ & OxA-26138 & Moccasin & -19.3 & & & & \\
\hline & $42 \mathrm{BO} 1$ & OxA-18159 & Moccasin & -19.4 & & & & \\
\hline & 5DA272 & \#1566 & Moccasin & -9.1 & -13.6 & 36.3 & 12.6 & 3.4 \\
\hline & 5DA272 & \#2805 & Sewn fragment & -11.9 & -16.4 & 44.0 & 15.8 & 3.2 \\
\hline & 5DA272 & \#3505 & Moccasin & -13.9 & -18.4 & 40.9 & 12.6 & 3.8 \\
\hline & 5DA272 & \#440 & Sewn fragment & -19.5 & -24.0 & 38.7 & 13.1 & 3.4 \\
\hline & 5DA272 & $\# 454$ & Sewn fragment & -19.5 & -24.0 & 35.9 & 12.6 & 3.3 \\
\hline & 5DA272 & \#455 & Leather strip & -19.6 & -24.1 & 44.6 & 14.9 & 3.5 \\
\hline \multicolumn{9}{|l|}{ Dung } \\
\hline & $42 \mathrm{BO} 1$ & FS-120 & & -26.0 & -25.0 & 36.7 & 1.1 & 38.1 \\
\hline & $42 \mathrm{BO} 1$ & FS-128 & & -24.9 & -23.9 & 36.0 & 1.0 & 41.1 \\
\hline & $42 \mathrm{BO} 1$ & FS-193 & & -26.5 & -25.5 & 31.6 & 1.0 & 37.0 \\
\hline
\end{tabular}


Table 1. Continued.

\begin{tabular}{|c|c|c|c|c|c|c|c|c|}
\hline Material & Site & Sample & Element/Type & $\delta^{13} \mathrm{C}_{\text {tissue }}$ & $\delta^{13} \mathrm{C}_{\text {diet }}$ & $\% \mathrm{C}$ & $\% \mathrm{~N}$ & $\mathrm{C} / \mathrm{N}$ \\
\hline & 42BO1 & FS-236 & & -26.7 & -25.7 & 35.3 & 1.2 & 33.5 \\
\hline & 42BO2 & FS-241 & & -24.7 & -23.7 & 31.6 & 1.0 & 38.0 \\
\hline & 42BO1 & FS-425 & & -24.4 & -23.4 & 35.6 & 1.1 & 38.8 \\
\hline & 42BO1 & FS-542 & & -25.1 & -24.1 & 33.9 & 1.4 & 29.1 \\
\hline & 42BO1 & FS-586 & & -23.2 & -22.2 & 39.3 & 1.0 & 46.7 \\
\hline & 42BO1 & FS-643 & & -26.7 & -25.7 & 31.0 & 1.0 & 37.4 \\
\hline & 42BO1 & FS-657 & & -25.8 & -24.8 & 37.8 & 0.9 & 48.8 \\
\hline & $42 \mathrm{BO} 2$ & FS-660 & & -25.7 & -24.7 & 40.7 & 1.0 & 46.5 \\
\hline & 42BO1 & FS-703 & & -25.6 & -24.6 & 36.4 & 1.0 & 43.8 \\
\hline & 42BO1 & FS-703 & & -24.1 & -23.1 & 35.2 & 1.6 & 25.3 \\
\hline & 42BO1 & FS-703 & & -22.1 & -21.1 & 36.9 & 1.4 & 30.6 \\
\hline & 42BO1 & FS-720 & & -25.6 & -24.6 & 35.6 & 1.1 & 39.4 \\
\hline & 42BO1 & FS-745 & & -25.8 & -24.8 & 36.8 & 1.0 & 41.5 \\
\hline & 42BO1 & FS-921 & & -25.2 & -24.2 & 35.8 & 0.9 & 45.6 \\
\hline & $\mathrm{AI}^{\mathrm{c}}$ & AN-447 & Collected dry & -28.2 & -25.1 & 39.6 & 1.4 & 33.6 \\
\hline & $\mathrm{AI}^{\mathrm{c}}$ & AN-448 & Collected dry & -26.8 & -23.7 & 36.3 & 1.2 & 36.2 \\
\hline & $\mathrm{AI}^{\mathrm{c}}$ & AN-449 & Collected dry & -27.0 & -23.9 & 27.3 & 1.4 & 23.0 \\
\hline & $\mathrm{AI}^{\mathrm{c}}$ & $\mathrm{AN}-450$ & Collected dry & -28.2 & -25.1 & 36.9 & 1.8 & 23.6 \\
\hline & $\mathrm{AI}^{\mathrm{c}}$ & AN-451 & Collected wet & -30.2 & -27.1 & 34.6 & 2.1 & 19.2 \\
\hline & $\mathrm{AI}^{\mathrm{c}}$ & AN-452 & Collected wet & -28.7 & -25.6 & 39.3 & 1.8 & 25.3 \\
\hline
\end{tabular}

Note: Diet $\delta^{13} \mathrm{C}$ values were calculated from measured tissue values using the equations in the text. An Excel version of this table is provided as Supplemental Table 1.

${ }^{\mathrm{a}}$ Values for these samples are means are from serial sampling (see Supplemental Table 2 for values of sections).

${ }^{\mathrm{b}}$ Carbon isotope values for these samples were obtained in the process of radiocarbon dating and previously published in Ives and colleagues (2014). Their diet values are not calculated because the calibration does not provide the necessary precision for paleodiet reconstruction.

${ }^{\mathrm{c}}$ Antelope Island diet calculations account for $\mathrm{a}+2.1 \%$ adjustment to make them equivalent to preindustrial values, as described in the text.

replicates were included in each run to monitor measurement uncertainty. For bone collagen, hair, hide, and dung (respectively), total analytical uncertainty was estimated to be $\pm 0.22 \%$, $\pm 0.18 \%$ o, $\pm 0.24 \%$ o, and $\pm 0.43 \%$ o (for error calculation method, see Szpak et al. 2017). As shown in Table 1 , consistent $\% \mathrm{C}, \% \mathrm{~N}$, and $\mathrm{C} / \mathrm{N}$ ratios indicate excellent preservation of collagen, hair, and hide.

Genetic analyses of the FS-305 ankle-wrap hide sample were conducted at the UCSC Paleogenomics Laboratory (PGL) to (1) obtain a taxonomic identification and (2) determine the sex of the animal. Further details of both isotopic and genetic methods can be found in Supplemental Text 1.

\section{Results and Discussion}

\section{Moccasin Leather Outlier: Context and Isotopic Composition}

The FS-305 sample, which produced the outlying $\delta^{13} \mathrm{C}$ value that inspired this study, came from an ankle wrap remnant on a fragmentary moccasin recovered from Promontory Cave 1 (Figure 3a). The ankle wrap is one of three separate pieces of leather, along with the body and vamp, used in the construction of Promontory-style moccasins (Figure 4). The FS-305 moccasin was missing the toe area, but its inferred size extrapolated from complete moccasins in the Promontory caves is about 22.5-24 $\mathrm{cm}$ (Erika Sutherland, personal communication 2019). Based on correlations between foot size, height, and age, this moccasin was probably worn by a youth approximately 11-13 years old (Billinger and Ives 2015).

The ankle wrap of FS-305 was originally selected as a convenient (minimally destructive) sampling location for an earlier AMS radiocarbon dating study. For that study, the sample underwent the Oxford Radiocarbon Accelerator Unit (ORAU)'s routine leather pretreatment process (lab code ACJ; Brock et al. 2010) and yielded an AMS date of 725 \pm 24 BP (OxA-25234), calibrated to $\mathrm{AD}$ 


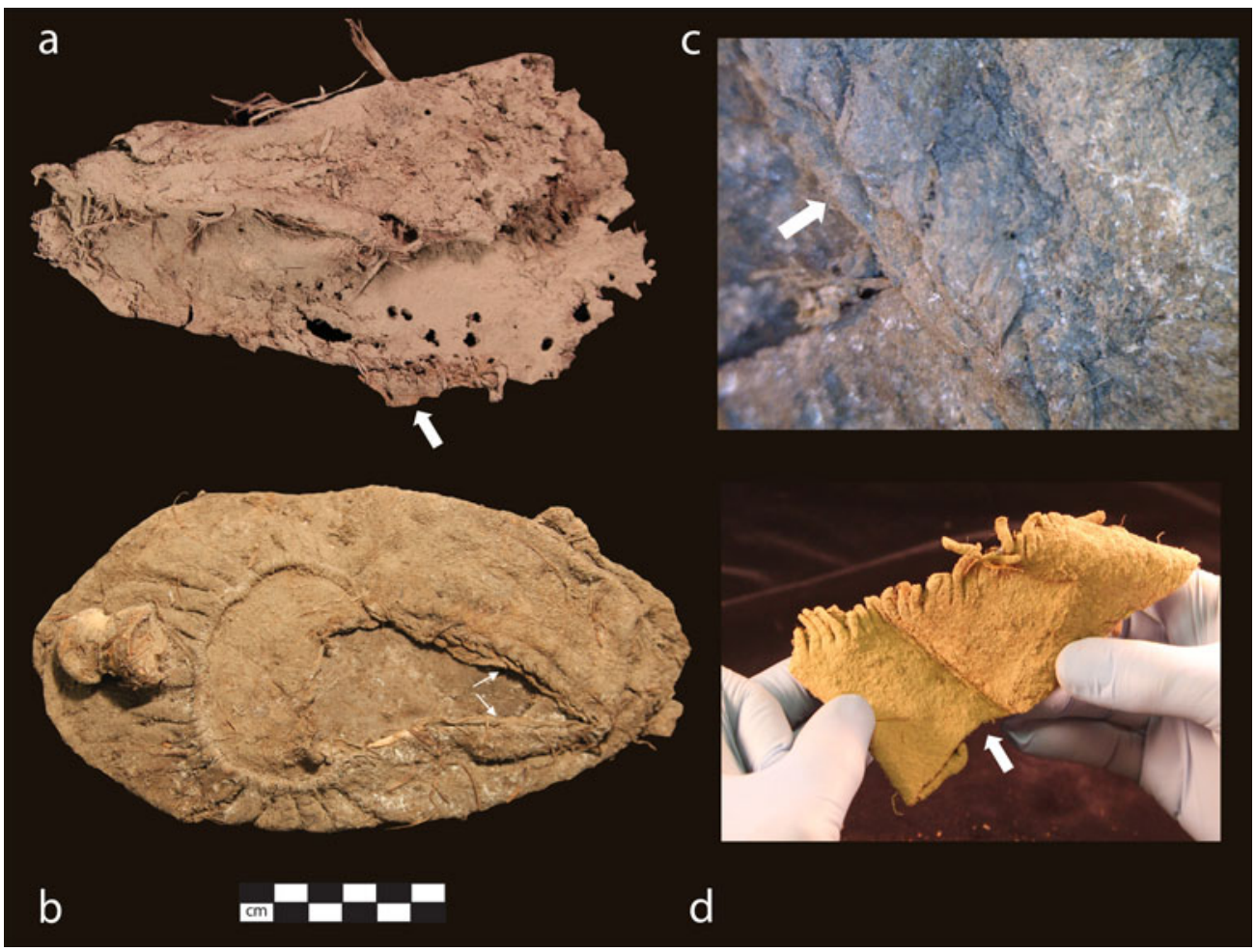

Figure 3. (a) Promontory Cave 1 partial moccasin FS-305, whose ankle wrap fragment (arrow), still stitched to the moccasin body, yielded an outlying $\delta^{13} \mathrm{C}$ value; (b) Promontory Cave moccasin FS-969, with arrows illustrating the location of cuts that severed its ankle wrap; (c) magnified image (30x) of the cut ankle-wrap surface (arrow) on moccasin FS-969, the remnant of which remained stitched to the moccasin body; (d) a fully severed ankle wrap from Promontory Cave 1 (artifact FS-238), with arrow indicating the cut edge. Photographs by J.W. Ives.

1252-1290 using OxCal v4 (Bronk Ramsey 2009) and the IntCal13 dataset (Reimer et al. 2013). Its associated $\delta^{13} \mathrm{C}$ was $-12.5 \%$ and its $\% \mathrm{C}, \% \mathrm{~N}$, and $\mathrm{C} / \mathrm{N}$ were $37 \%, 14 \%$, and 3.2 , respectively. In the present study, a new aliquot of the same sample was independently cleaned as described in the Supplemental Text 1, and its isotopic composition was obtained following a 2-point calibration that produced the accuracy and precision necessary for paleodietary analysis (Szpak et al. 2017). Duplicate analyses of FS-305 resulted in $\delta^{13} \mathrm{C}$ values of $-12.93 \%$ and $-12.87 \%$, carbon contents of $35.0 \%$ and $34.7 \%$, nitrogen contents of $12.3 \%$ and $12.5 \%$, and $\mathrm{C} / \mathrm{N}$ ratios of 3.32 and 3.24 (means listed in Table 1). The qualitycontrol indicators and reproducibility both within and between labs indicate excellent preservation and support the validity of the isotopic measurements. Converting the mean of the measured $\delta^{13} \mathrm{C}$ value obtained from the leather $\left(-12.9 \%\right.$ ) to a diet $\delta^{13} \mathrm{C}$ value results in an estimate of $-17.4 \%$ for the plants consumed by the animal while the skin was being formed.

After confirming the ankle wrap's unusual $\delta^{13} \mathrm{C}$, we tested the body portion of the same moccasin. The FS-305 moccasin body leather produced a $\delta^{13} \mathrm{C}$ value of $-20.0 \%$, which results in a dietary $\delta^{13} \mathrm{C}$ estimate of $-24.5 \%$ (Table 1). The moccasin body $\delta^{13} \mathrm{C}$ is distinctly different from that of the ankle wrap but is consistent with all 171 other northern Utah bison samples (Table 1).

\section{Local Bison Eating $C_{4}$ and/or CAM Plants?}

The thickness and general appearance of the FS-305 moccasin leather were consistent with bison. Our genetic analysis supports this species attribution (see Supplemental Text 1). 


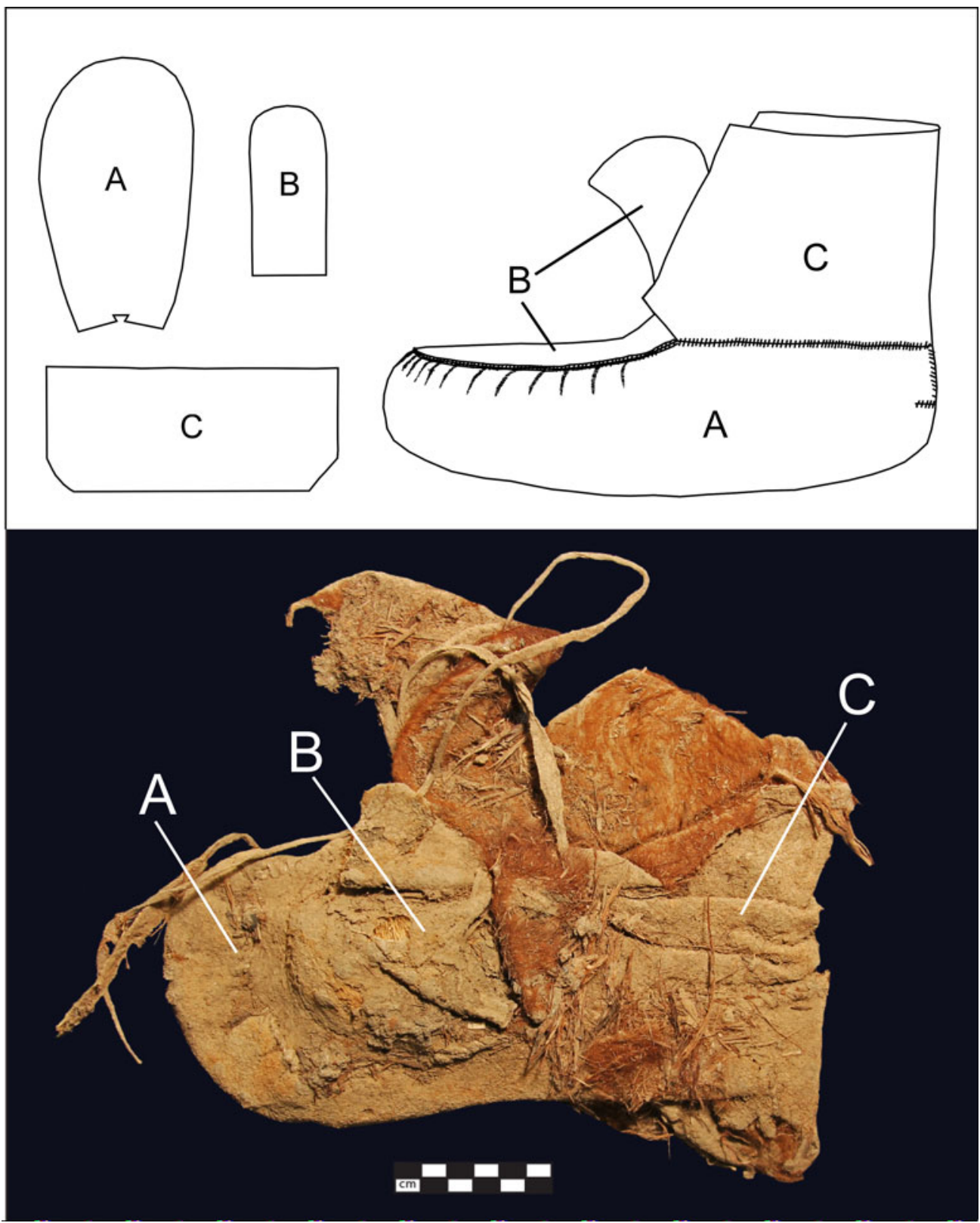

Figure 4. Promontory-style moccasins are constructed from three separate pieces of leather: (a) the body, including the sole; (b) the vamp, and (c) the ankle wrap. Top: A Promontory-style (BSM 2 [Bb]) moccasin pattern based on the complete Franktown example (\#3505). Bottom: A Promontory Cave 1 moccasin (UMNH.A.8011.18, FS-42BO1.801.1) with bison-fur lining and ankle wrap still attached. Drawing by K.P. Gilmore. Photograph by J.W. Ives.

The FS-305 ankle wrap was the only northern Utah bison tissue sample with clear evidence for $\mathrm{C}_{4} / \mathrm{CAM}$ plant consumption. All of the other carbon isotope measurements for Promontory bison tissues are consistent with purely $\mathrm{C}_{3}$ diets
(Table 1; Figure 5). Even bison dung, which would be most likely to record short-term dietary deviations from the norm, clearly reflects a $\mathrm{C}_{3}$ diet. (We note that a single dung sample has a $\delta^{13} \mathrm{C}$ value $0.3 \%$ o higher than our $\mathrm{C}_{3}$ cutoff, but 
because analytical uncertainty for dung was $\pm 0.43 \%$, this sample does not provide solid evidence of $\mathrm{C}_{4}$ plant consumption.) Serially sampled Promontory bison hair, including several long hairs that likely record at least a year of growth, show no evidence of temporary shortterm consumption of $\mathrm{C}_{4}$ or CAM plants. Instead, each individual hair shows variations of $\leq 1.8 \%$, all of which fall firmly within the $\mathrm{C}_{3}$ range (Figure 6; Supplemental Table 2). Mediumand long-term bison diet records, including the bone collagen and hide/leather values, also show no evidence of $\mathrm{C}_{4}$ or CAM plant consumption (Table 1; Figure 5). Fourteen modern Antelope Island bison dung and hair samples further show no evidence of $\mathrm{C}_{4}$ or CAM plant consumption along the shores of Great Salt Lake (Table 1; Figure 5).

The FS-305 leather sample clearly has a much higher $\delta^{13} \mathrm{C}$ value than any of the other Promontory samples (Figure 5). In contrast to the abundant evidence that local Promontory bison diets were entirely $\mathrm{C}_{3}$, the FS-305 ankle wrap has a $\delta^{13} \mathrm{C}$ value that indicates the animal consumed a large amount of $\mathrm{C}_{4}$ (and/or CAM) plants. A linear $\% \mathrm{C}_{4}$ estimate can provide a general approximation of the relative amount of $\mathrm{C}_{4}$ versus $\mathrm{C}_{3}$ plants that would have had to have been consumed to produce a particular $\delta^{13} \mathrm{C}$ value. If we assume a pure $\mathrm{C}_{3}$ dietary endpoint of $-24.3 \%$ o (the mean derived from typical Promontory bison values obtained in this study) and a pure $\mathrm{C}_{4} / \mathrm{CAM}$ endpoint of $-10.3 \%$ o (the mean of ancient Colorado Plateau CAM plants, which overlap with $\mathrm{C}_{4}$ plants; Metcalfe and Mead 2019), the FS-305 diet value of $-17.4 \%$ o suggests a $\mathrm{C}_{4} / \mathrm{CAM}$ contribution of approximately $50 \%$. For roughly $50 \%$ $\mathrm{C}_{4} / \mathrm{CAM}$ to be recorded in a relatively slowforming tissue such as skin, the $\mathrm{C}_{4}$ and/or CAM plants must have comprised a significant proportion of the diet for several weeks to months. Yet, there is no unequivocal evidence for $\mathrm{C}_{4}$ or CAM plant consumption in any other Promontory bison tissue, even those that formed over short timescales and would therefore record brief temporary changes in diet. We conclude that the FS-305 sample came from a bison that was not native to the Promontory region.

\section{Wandering Bison?}

Could the outlying $\delta^{13} \mathrm{C}$ of the FS-305 ankle wrap be the result of a bison migrating into the Promontory area after spending an earlier part of its life in a high- $\mathrm{C}_{4}$ grass area? As demonstrated by the $\mathrm{C}_{3}$ signals obtained from 171 samples of Promontory and Antelope Island bison dung, hair, hide, and bone collagen, this would have been a highly unusual occurrence. But if it did occur, what locations would have had sufficiently high grass $\delta^{13} \mathrm{C}$ values to produce the FS-305 value?

Cotton and colleagues (2016) predictively modeled the distributions of $\mathrm{C}_{3} / \mathrm{C}_{4}$ grasses and mean grass $\delta^{13} \mathrm{C}$ values across modern (preindustrial) North America. Given that this model was based in part on measured bison $\delta^{13} \mathrm{C}$ values that were not included in the present study, it provides an excellent baseline for determining possible regions of bison origin (Figure 7). Local Promontory bison $\operatorname{diet} \delta^{13} \mathrm{C}$ estimates from dung, hair, hide, and bone collagen are in good agreement with one another and with the predicted values for the Promontory region (Table 2; Figure 7). The nearest areas on the grass isoscape with mean $\delta^{13} \mathrm{C}$ values similar to that of the FS-305 bison are about $500 \mathrm{~km}$ to the south, in southwestern Utah / northwestern Arizona, and about $700 \mathrm{~km}$ east, around Franktown Cave in Colorado. We cannot exclude the possibility that closer locations might include microhabitats with high $\mathrm{C}_{4}$, particularly during the summer when $\mathrm{C}_{4}$ grasses flourish. It is clear from our baseline isotopic data, however, that if such areas existed near the caves, local Promontory bison did not utilize them.

To determine whether a bison could feasibly have traveled under its own power from one of these faraway locations to Promontory while retaining a strong $\mathrm{C}_{4}$ signal in its hide, we consider the distance traveled (estimated using a least-cost path approach), the isotopic turnover rate of skin (because continuous replacement would gradually "dilute" the $\mathrm{C}_{4}$ signal as an animal switched its diet during the journey), and the feasibility of traveling the required distance in the required time (based on modern bison observational studies). Supplemental Text 2 provides details of these inferences. We conclude that it may have been physically possible 


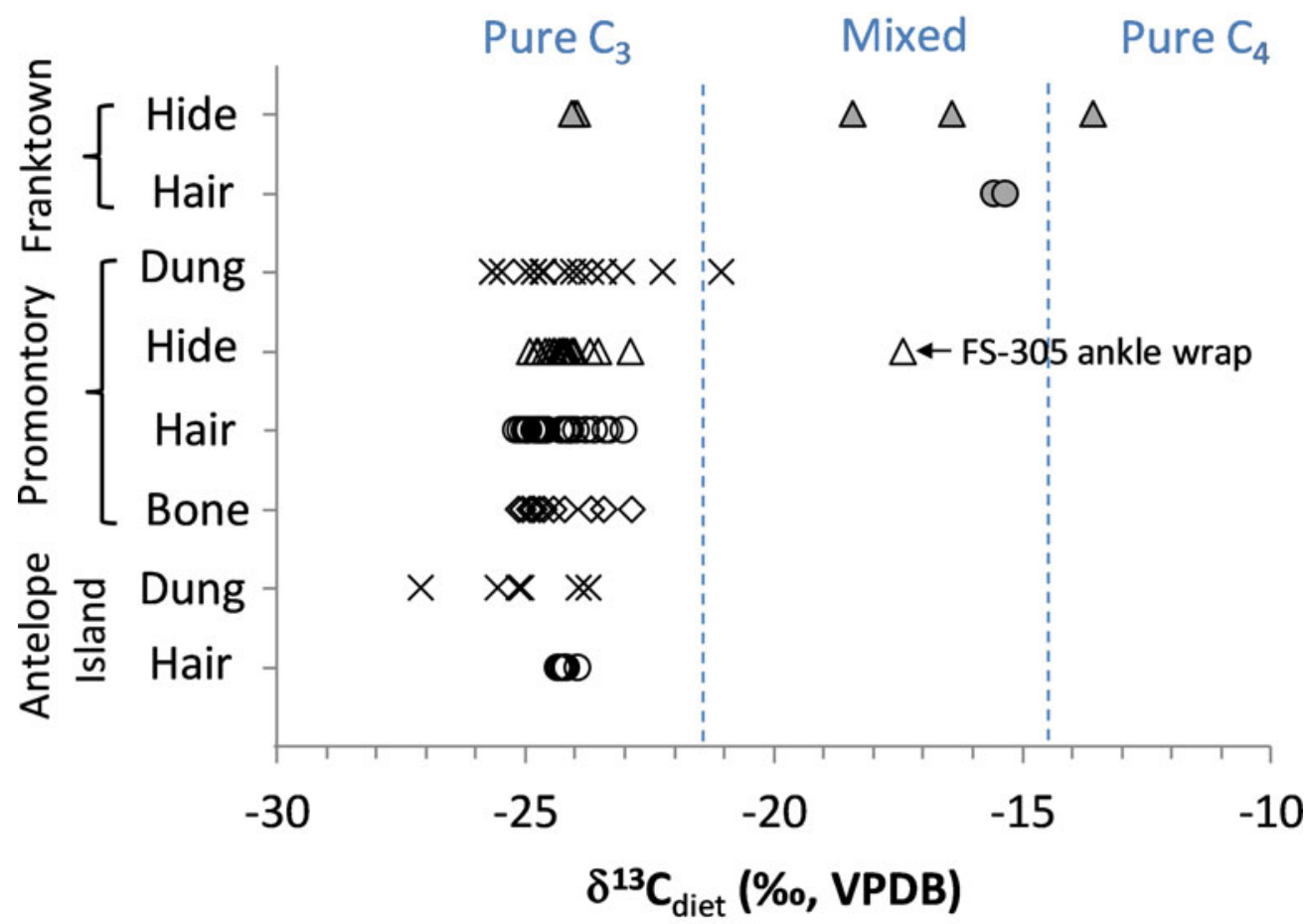

Figure 5. Comparison of diet $\delta^{13} \mathrm{C}$ values calculated from measured tissue values of Promontory-era bison recovered from Promontory and Franktown caves, and modern bison from Antelope Island. Tissue $\delta^{13} \mathrm{C}$ values were converted to diet $\delta^{13} \mathrm{C}$ values using the equations provided in the text. Modern Antelope Island $\delta^{13} \mathrm{C}$ values were further adjusted by $2.1 \%$ to account for the Suess Effect. Filled symbols are samples from Franktown Cave. Open symbols are samples from northern Utah (Promontory or Antelope Island). Triangles indicate values derived from hide; crosses, from dung; circles, from hair; and diamonds, from bone.

for a bison to travel those distances in the required amount of time, but it would have been highly unlikely-particularly because genetic analyses indicates that the leather was from a female bison, which would typically travel with calves (Supplemental Texts 1 and 2). Altogether, it is unlikely that the FS-305 ankle wrap came from a wandering bison.

\section{Procurement of Hide via Trade?}

If the FS-305 ankle wrap leather was not from a local bison or a bison wanderer, then it must have been procured in a faraway location and transported to Promontory Cave 1. It is possible that the FS-305 ankle wrap leather was obtained through trade. In this scenario, the Promontory people would have obtained the leather from other individuals or groups who had been in a location far to the south or east (or who had traded for bison leather from these areas).
However, based on the ready availability of local bison to Promontory people, we believe that trade in bison products was very unlikely. In a less than $3 \mathrm{~m}^{2}$ area of Cave 1, more than 30,000 whole and fragmentary bones were recovered. These are completely dominated by large game animals, with bison the most common genus for identifiable specimens (Ives, Johansson, et al. 2014; Johansson 2013). The presence of large, difficult-to-transport skulls and low-utility lower limbs and hooves suggest that a kill site was nearby. Indeed, the landscape above the caves funnels prey into a narrow area that is ideal for ambush strategies and remains an active game trail today. Furthermore, Promontory Cave 1 occupants were not heavily comminuting bones to extract greases as they would if food were scarce. Strata consisting of charred and calcined bone burned at variable temperatures suggest that greases and fats were present 


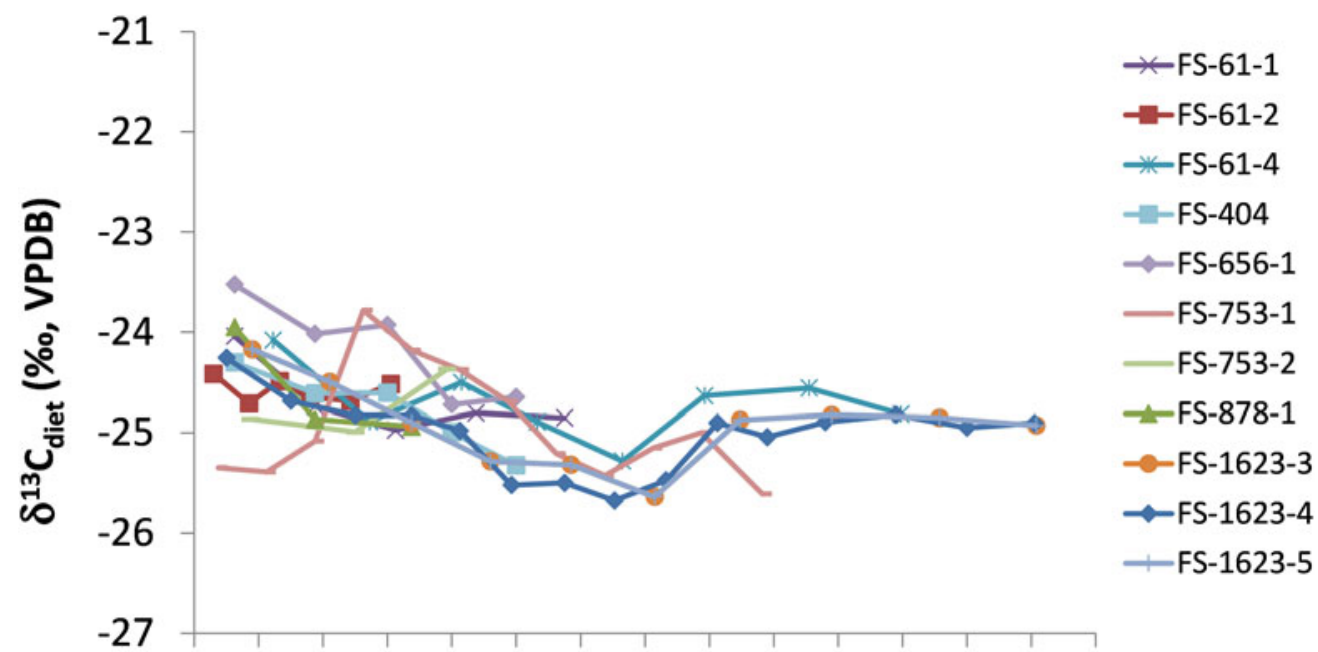

\section{$\begin{array}{lllllllllllllll}0 & 2 & 4 & 6 & 8 & 10 & 12 & 14 & 16 & 18 & 20 & 22 & 24 & 26 & 28\end{array}$}

\section{Distance from root or base $(\mathrm{cm})$}

Figure 6. Diet $\delta^{13} \mathrm{C}$ values (calculated from measured hair values, as described in the text) of 11 individual Promontory bison hairs that were serially sampled to examine changes over time. Diet $\delta^{13} \mathrm{C}$ values less than $-21.4 \%$ are within the range of purely $\mathrm{C}_{3}$ diets, as described in the text.

and served as accelerants for a fire rather than being extracted for food (Ives, Johansson, et al. 2014). Based on the number of discarded moccasins in the caves, Reilly (2015) estimated that about 250 bison hides would have been required to replace them. In combination with the skeletal evidence, this suggests that the minimum number of bison individuals (MNI) for the entire cave contents would be huge. Thus, multiple lines of evidence indicate that bison were abundant around the Promontory caves and that the Promontory people were highly adept at hunting them. It would have been highly unnecessary for Promontory people to trade for bison leather. Although we cannot completely exclude this possibility, we consider the explanation in the next section more likely.

\section{Procurement of Hide via Long-Distance Travel}

The final and likeliest possibility to explain how the FS-305 ankle wrap leather ended up incorporated into a Promontory Cave 1 moccasin is longdistance travel to/from an area far to the south or east. Below, we will first explore potential areas of origin and then discuss possible mechanisms of transportation, including a long-distance round trip, a meeting with individuals from distant regions at an intermediate location, or a visit from outsiders who left behind the hide or leather.

It is not possible to pinpoint a precise location of origin for the FS-305 ankle wrap leather because its $\delta^{13} \mathrm{C}$ value of $-17.4 \%$ is characteristic of a vast region from South Dakota through Nebraska, eastern Colorado, southeastern New Mexico, and southern Arizona (Figure 7). We also cannot exclude the possibility that the bison inhabited a microhabitat with high $\mathrm{C}_{4}$ some distance from the caves but closer than the areas identified in our regional isoscape. Based on our current knowledge, however, northwestern Arizona and central Colorado are the closest locations to have $\delta^{13} \mathrm{C}$ values consistent with that of the FS-305 ankle wrap (Figure 7).

The northwestern Arizona region with modeled $\delta^{13} \mathrm{C}$ values consistent with the FS-305 ankle wrap sample is known to have supported bison during the Late Prehistoric period (Martin et al. 2017). Open-air sites with Promontory material culture associations dating approximately 100-200 years later than the Promontory 


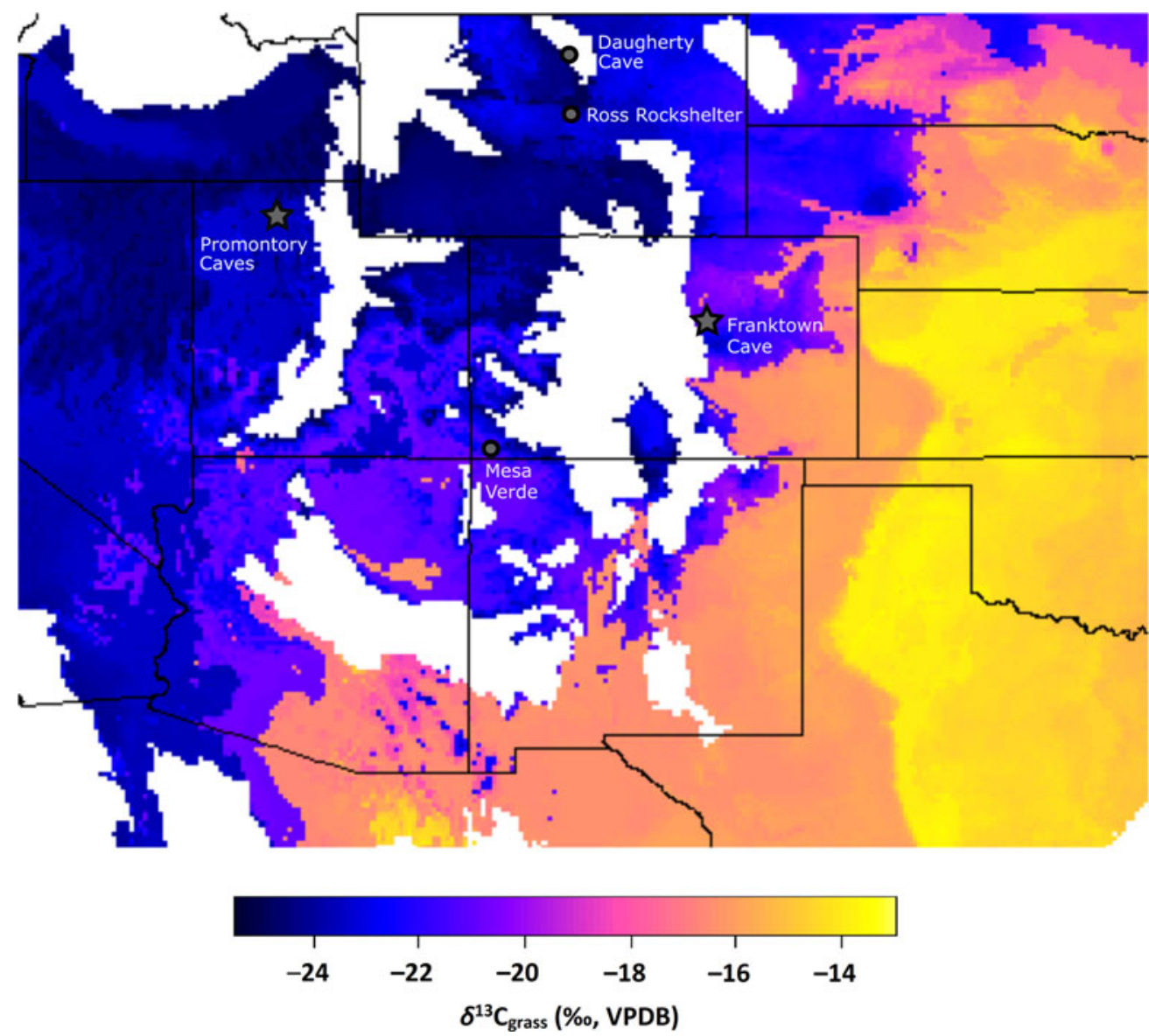

Figure 7. Modeled mean $\delta^{13} \mathrm{C}$ values of Late Prehistoric grasses based on previous studies of bison and mammoth $\delta^{13} \mathrm{C}$ and nine climatic variables, including growing season precipitation and temperature (adapted from Cotton et al. 2016). Areas in white have few grasses of any type and are therefore not included in the model. Stars indicate the locations of the Promontory caves and Franktown Cave. Circles indicate other locations with Promontory-style moccasins, as described in the text.

Table 2. Carbon Isotope Compositions (means \pm one standard deviation) of Promontory Caves and Antelope Island State Park Bison Tissues.

\begin{tabular}{llccccc}
\hline Location & Tissue & $\mathrm{N}$ & $\delta^{13} \mathrm{C}_{\text {tissue }}$ & $\delta^{13} \mathrm{C}_{\text {diet }}$ & $\Delta^{13} \mathrm{C}_{\mathrm{t}-\mathrm{d}}{ }^{\mathrm{a}}$ & $\Delta^{13} \mathrm{C}_{\text {Suess }}{ }^{\mathrm{b}}$ \\
\hline Promontory & Bone collagen & 12 & $-19.4 \pm 0.7$ & $-24.4 \pm 0.7$ & -5.0 & 0.0 \\
Promontory & Hair $^{\mathrm{c}}$ & 33 & $-21.4 \pm 0.5$ & $-24.4 \pm 0.5$ & -3.0 & 0.0 \\
Promontory & Hide $^{\mathrm{d}}$ & 18 & $-19.7 \pm 0.5$ & $-24.2 \pm 0.5$ & -4.5 & 0.0 \\
Promontory & Dung & 17 & $-25.2 \pm 1.2$ & $-24.2 \pm 1.2$ & +1.0 & 0.0 \\
Antelope Island & Hair & 8 & $-23.3 \pm 0.1$ & $-24.2 \pm 0.1$ & -3.0 & +2.1 \\
Antelope Island & Dung & 6 & $-28.2 \pm 1.2$ & $-25.1 \pm 1.2$ & +1.0 & +2.1 \\
\hline
\end{tabular}

${ }^{\mathrm{a}}$ Carbon isotope offset used to convert tissue to diet values, as described in the text.

${ }^{\mathrm{b}}$ Modern (Antelope Island) diet values were converted to preindustrial equivalents by adding $+2.1 \%$, as described in the text. ${ }^{c}$ Serially sampled hair results were averaged to produce a single value for each individual hair before being included here. ${ }^{\mathrm{d}}$ Includes only data obtained in the present study, using a 2-point calibration suitable for paleodiet reconstruction, as described in the text. 
cave deposits occur on the eastern shore of Great Salt Lake and Utah Lake (Janetski and Smith 2007), along the least-cost path route to this part of northwestern Arizona (Supplemental Text 2). With the cessation of Fremont maize agriculture around AD 1150 (Coltrain and Leavitt 2002), travel along the Wasatch Front and into northwestern Arizona during the late thirteenth century may have been relatively easy. Given that later Promontory Phase assemblages are situated in geographically intermediate areas, it would not be surprising if late thirteenth-century Promontory cave occupants had connections with regions as far south as northern Arizona. We note that Promontory-style moccasins have recently been identified near Mesa Verde (Figure 1), supporting the idea of a connection with the Southwest (Fewkes 1909; Osborne 2004; Yanicki and Ives 2017).

Alternatively, the isoscape model and Franktown Cave bison $\delta^{13} \mathrm{C}$ values are consistent with a potential origin for the FS-305 ankle wrap leather in central Colorado. Franktown bison hide and hair samples had highly variable $\delta^{13} \mathrm{C}$ values, ranging from purely $\mathrm{C}_{3}$ to purely $\mathrm{C}_{4}$ (Figure 5). The much larger variability in bison $\delta^{13} \mathrm{C}$ at Franktown compared to Promontory reflects the site's location in an area that rapidly transitions from $\mathrm{C}_{3}$ - to $\mathrm{C}_{4}$-dominated grassland (Figures 5 and 7) - that is, all of the Franktown bison could have been procured relatively close to Franktown Cave, with higher values among bison who had grazed in areas further east and/ or were killed after the summer months. The Promontory Cave FS-305 moccasin leather may therefore be evidence of contact between northern Utah and central Colorado. The assemblage similarities between the Promontory and Franktown caves support the possibility of contact in various forms: travel between the two locations, a rendezvous at a geographically intermediate location, or a rendezvous with yet another population that shared this material culture heritage. We note that Promontory-style moccasins are also present in Daugherty Cave (48WA302) and Ross Rockshelter (48NA331) in Wyoming, which are intermediate in longitude (although at higher latitudes) relative to Promontory and Franktown (Figures 1 and 7; Frison 1968; Garling 1964). ${ }^{1}$
It is significant that the only extralocal leather identified to date in the Promontory caves was used to construct a moccasin ankle wrap. Unlike moccasin bodies-which quickly become worn, patched, further worn, and discarded-ankle wraps can be reused and recycled into new footwear. Within the Promontory caves assemblage, the great majority of moccasins are heavily worn, patched, or both. Cuts that indicate the severing of ankle wraps from the moccasin body are common (Figure 3b-d). Repurposing of leather also occurred at Franktown Cave, where three fringed-seam artifacts dating to the Promontory occupation had been cut from larger leather objects (Gilmore et al. 2021). Although some leather scraps occur in both the Promontory and Franktown caves, their occupants were frugal, generally discarding only worn-out moccasin bodies and bison robe edges. Given its longer use life and potential for reuse, an ankle wrap is the one portion of a moccasin most likely to survive a long-distance journey, precisely because it could be usefully reincorporated into new footwear.

Historic sources show that it was essential to carry and repair additional moccasins during a lengthy journey. The distances to origin documented here would require wearing and replacing several pairs of moccasins, which were common discards in abandoned camps (e.g., Ewers 1955:182, 184; Moulton 1987:216, 219; Wilson 1924:212, 232, 283-284). We consider it plausible that the leather was obtained at a distant source and recycled in a series of usages as an ankle wrap over the course of the journey to the Promontory caves. It is also possible that the FS-305 ankle wrap leather was brought to the caves as a piece of clothing, bison robe, or lodge covering that was repurposed (although the larger the original object, the greater the possibility of detecting other anomalous values, which we have not). As discussed above, however, obtaining such items from other groups or individuals would have been like carrying coals to Newcastle. ${ }^{2}$ The Promontory cave occupants had access to a genuine abundance of bison products, including fine leather, and they would have had no need to acquire it from others while residing in the caves. It therefore seems unlikely that a visitor to the caves would leave behind leather 
that would later become incorporated into a Promontory moccasin. Instead, it seems more likely that the leather was obtained at some distance from the caves and reincorporated into the moccasin during travel. This implies that Promontory people had either traveled to one of these faraway locations themselves or met with others at an intermediate location where the leather changed hands. Either way, the ankle wrap came from far away and was not obtained in the vicinity of the caves, which implies long-distance connections and knowledge of faraway locations.

\section{Independent Evidence for Long-Distance Connections of Promontory Caves Inhabitants}

Although the ankle wrap $\delta^{13} \mathrm{C}$ value is a unique piece of evidence for human movement, it is far from the only evidence for long-distance connections of Promontory people. In addition to the Promontory material culture evidence at Franktown Cave and Promontory's northern artifact associations, gaming and obsidian source studies also provide support for longdistance interactions. The Promontory caves inhabitants were familiar with a diversity of games whose distributions span much of western North America, suggesting a wide range of social interactions and possibly longdistance movements (Yanicki and Ives 2017). $\mathrm{X}$-ray fluorescence indicates that the majority of obsidian artifacts recovered from Promontory Caves 1 and 2 were constructed from Malad obsidian sourced in southern Idaho (Hughes 2011, 2012). Around the time of the Promontory and Franktown cave occupations, material from Malad and other northern obsidian sources increased by an order of magnitude on the central and southern Plains (Gilmore, Hedlund, and Clark 2017; Hoard et al. 2008; Hughes et al. 2019). Promontory groups may have been involved in the distribution of Malad obsidian through direct migration, down-the-line trade, or trade involving more distant redistributive centers. Whichever alternatives were involved, the late-period obsidian distribution and gaming evidence support the hypothesis that Promontory Phase communities participated in long-distance interaction spheres from north of Utah far to the south and east.

\section{Implications for Promontory Lifeways}

For hunter-gatherer groups, Seymour (2012:378) recommends reserving the term "migration" to describe movement to a new region that was not previously utilized, and "mobility" to describe movements within regularly used territories. Given the fact that only one Promontory caves specimen has been discovered with a $\mathrm{C}_{4}$ isotopic signal, it is unlikely that regular Promontory mobility encompassed regions with abundant $\mathrm{C}_{4}$ grasses. Several lines of evidence support geographically widespread interactions, however, and the isotopic evidence suggests that at least one long-distance voyage was likely made. Anthony (1990) has identified categories of long-distance activities associated with migration that may have detectable material correlates: scouting (to facilitate long-distance "leapfrog" migrations), return visits to a previous occupation, long-distance movements for seasonal or task-specific purposes, stream migration (movement of successive groups along a well-defined route), and counterstreaming (returning to a former occupation after migrating away). If Promontory people had traveled to a faraway location, long-distance scouting seems the most plausible purpose for such a voyage. Establishing familiarity with distant regions would be an essential strategy for highly mobile groups infiltrating the changing thirteenth-century social landscape of the American Southwest and central/southern Plains. Scouting for new economic, trading, or raiding opportunities could have served as a precursor to a shift in regularly used territories, equipping the Promontory people with broad landscape knowledge that could be mobilized to facilitate migration (Seymour 2012). The latter seems to have occurred at the end of the thirteenth century, when Promontory people ceased intensive occupation of the Promontory caves (Ives, Froese, et al. 2014).

\section{Conclusions}

Exceptional organic preservation allowed us to establish a uniquely strong isotopic baseline for bison in the area around the Promontory caves during the late thirteenth century. Analysis of 171 bison samples (dung, hair, hide, bone) that 
formed over short, medium, and long timescales suggests that Promontory bison consumed purely $\mathrm{C}_{3}$ diets. In contrast, the ankle wrap of one Promontory moccasin was from a bison that consumed a large amount of $\mathrm{C}_{4}$ plants over a period of several weeks or months. Based on the local Promontory isotopic baseline along with our current knowledge of skin turnover rates and $\mathrm{C}_{4}$ grass distributions, we argued that it was unlikely the leather came from either a bison with an unusual local diet or a bison that migrated to Promontory from a distant location. Instead, this animal was killed far to the east or south of the Promontory caves. We argued that trade in bison leather was unlikely given that bison were abundant in areas around the caves. Therefore, we consider long-distance travel to be the likeliest explanation, with a scrap of leather procured in the south/east recycled into an ankle wrap over the course of the journey, as would have been common during long-distance voyages. Possible source regions for the ankle wrap leather include northwestern Arizona, central Colorado, and areas farther to the south and east. Regardless of the precise location, the evidence suggests a connection with an area far from the Promontory caves. Knowledge of distant locations, whether obtained through long-distance scouting or contact with other groups, would have ensured that Promontory people were well prepared for relocation to a new "home territory" when the need arose. It appears that this occurred at the end of the thirteenth century, when intensive occupation of the caves ceased (Ives, Froese, et al. 2014).

This study has demonstrated an approach for investigating long-distance connections that is viable for organic remains in regions with minimal local isotopic variability (i.e., $\mathrm{C}_{3}$-dominated grasslands), where the local isotopic baseline is well constrained and considerably different from potential extralocal source regions. This approach is not useful for areas with highly variable local plant $\delta^{13} \mathrm{C}$ values (i.e., abundant $\mathrm{C}_{3}$ and $\mathrm{C}_{4}$ plants), such as Franktown Cave. Crucial to our approach was the availability of different "time records" from animal tissues preserved in dry cave contexts (dung, hair, hide, bone), and the integration of multidisciplinary lines of evidence (isotopic, genetic, ecological, archaeological). We echo Seymour's (2010) argument that "anomalous" data can be sources of valuable information rather than errors to be averaged away, especially for highly mobile populations.

Acknowledgments. We thank Paul Stavast, Vandy Bowyer, Harvey Friebe, Evangeline Bell, and Amy Sky for sampling and laboratory assistance; Jennifer Cotton for access to isoscape data; Kathy Lemberg, Gabriel Bowen, and Rhy McMillan for map assistance; Joel Janetski for manuscript comments; Steven Bates for permission to sample in Antelope Island State Park; and Wes Olson, Johan du Toit, and Tom Seaton for insights into bison ecology. This research was funded by a Social Sciences and Humanities Research Council of Canada (SSHRC) Banting Postdoctoral Fellowship (awarded to JZM), and by SSHRC Standard Research Grant 410-2010-0480 and SSHRC Insight Grant 435-20120140 (awarded to JWI). We are grateful to landowners George and Kumeroa Chournos for the opportunity to conduct research in the Promontory caves.

Data Availability Statement. Isotopic data presented in this article are available in the Supplemental Materials associated with this manuscript. Genetic data are available on the online data storage platform Dryad (DOI:10.7291/D18Q23).

Supplemental Materials. For supplemental materials accompanying this article, visit https://doi.org/10.1017/aaq.2020. 116.

Supplemental Text 1. Supplemental materials and methods. Supplemental Text 2. Supplemental discussion (wandering bison).

Supplemental Table 1. Excel version of Table 1.

Supplemental Table 2. Results from serially sampled Promontory Caves bison hair.

\section{Notes}

1. In a forthcoming publication, we will provide details of the Daugherty Cave and Ross Rockshelter moccasins, including their dating and association with artifacts that include incised cane dice, incised stone tablets, and moccasin pattern cutouts as also occur in the Promontory caves.

2. Newcastle was long a major British center for coal mining, leading to this usage as an idiom for a redundant or pointless action.

\section{References Cited}

Achilli, Alessandro, Ugo A. Perego, Hovirag Lancioni, Anna Olivieri, Francesca Gandini, Baharak H. Kashani, Vincenza Battaglia, Viola Grugni, Norman Angerhofer, Mary P. Rogers, Rene J. Herrera, Scott R. Woodward, Damian Labuda, David G. Smith, Jerome S. Cybulski, Ornella Semino, Ripan S. Malhi, and Antonio Torroni

2013 Reconciling Migration Models to the Americas with the Variation of North American Native Mitogenomes. PNAS 110:14308-14313.

Alves-Stanley, Christy D., and Graham A. J. Worthy

2009 Carbon and Nitrogen Stable Isotope Turnover Rates and Diet-Tissue Discrimination in Florida Manatees 
(Trichechus manatus latirostris). Journal of Experimental Biology 212:2349-2355.

Ambrose, Stanley H.

1990 Preparation and Characterization of Bone and Tooth Collagen for Isotopic Analysis. Journal of Archaeological Science 17:431-451.

Ames, Audra L., Edward S. Van Vleet, and William M. Sackett

1996 The Use of Stable Carbon Isotope Analysis for Determining the Dietary Habits of the Florida Manatee, Trichechus manatus latirostris. Marine Mammal Science 12:555-563.

Anthony, David W.

1990 Migration in Archeology: The Baby and the Bathwater. American Anthropologist 92:895-914.

Arkush, Brooke S.

2016 Late Prehistoric Big-Game Hunting in Curlew Valley: Archaeological Investigations at 10OA275. Monographs in Idaho Archaeology and Ethnology No. 2. Archaeological Survey of Idaho, Idaho State Historical Society, Boise.

Benson, Larry V., Michael S. Berry, Edward A. Jolie, Jerry D. Spangler, David W. Stahle, and Eugene M. Hattori

2007 Possible Impacts of Early 11th-, Middle 12th-, and Late 13th-Century Droughts on Western Native Americans and the Mississippian Cahokians. Quaternary Science Reviews 26:336-350.

Bentley, R. Alexander, and Corina Knipper

2005 Geographical Patterns in Biologically Available Strontium, Carbon and Oxygen Isotope Signatures in Prehistoric SW Germany. Archaeometry 47:629-644.

Billinger, Michael, and John W. Ives

2015 Inferring Demographic Structure with Moccasin Size Data from the Promontory Caves, Utah. American Journal of Physical Anthropology 156:76-89.

Bocherens, Hervé, Aurora Grandal-d'Anglade, and Keith A. Hobson

2014 Pitfalls in Comparing Modern Hair and Fossil Bone Collagen $\mathrm{C}$ and $\mathrm{N}$ Isotopic Data to Reconstruct Ancient Diets: A Case Study with Cave Bears (Ursus spelaeus). Isotopes in Environmental and Health Studies 50:291-299.

Brock, Fiona, Thomas Higham, Peter Ditchfield, and Christopher Bronk Ramsey

2010 Current Pretreatment Methods for AMS Radiocarbon Dating at the Oxford Radiocarbon Accelerator Unit (ORAU). Radiocarbon 52:103-112.

Bronk Ramsey, Christopher

2009 Bayesian Analysis of Radiocarbon Dates. Radiocarbon 51:337-360.

Browning, Nicole E., Christopher Dold, Jack I-Fan, and Graham A. J. Worthy

2014 Isotope Turnover Rates and Diet-Tissue Discrimination in Skin of ex situ Bottlenose Dolphins (Tursiops truncatus). Journal of Experimental Biology 217:214 221.

Cerling, Thure E., John M. Harris, and Meave G. Leakey 1999 Browsing and Grazing in Elephants: The Isotope Record of Modern and Fossil Proboscideans. Oecologia 120:364-374.

Clark, William Philo

1885 The Indian Sign Language: With Brief Explanatory Notes of the Gestures Taught Deaf-Mutes in Our Institutions for Their Instruction, and a Description of Some of the Peculiar Laws, Customs, Myths, Superstitions, Ways of Living, Code of Peace and War Signals of Our Aborigenes. L. R. Hamersly, Philadelphia.
Coltrain, Joan Brenner, and Steven W. Leavitt

2002 Climate and Diet in Fremont Prehistory: Economic Variability and Abandonment of Maize Agriculture in the Great Salt Lake Basin. American Antiquity 67:453-485.

Cotton, Jennifer M., Thure E. Cerling, Kathryn A. Hoppe, Thomas M. Mosier, and Christopher J. Still

2016 Climate, $\mathrm{CO}_{2}$, and the History of North American Grasses since the Last Glacial Maximum. Science Advances 2(3):e1501346. DOI:10.1126/sciadv.1501346.

Crowley, Brooke E., Melinda L. Carter, Sarah M. Karpanty, Adrienne L. Zihlman, Paul L. Koch, and Nathaniel J. Dominy

2010 Stable Carbon and Nitrogen Isotope Enrichment in Primate Tissues. Oecologia 164:611-626.

Drucker, Dorothée G., Anne Bridault, Keith A. Hobson, Elwira Szuma, and Hervé Bocherens

2008 Can Carbon-13 in Large Herbivores Reflect the Canopy Effect in Temperate and Boreal Ecosystems? Evidence from Modern and Ancient Ungulates. Palaeogeography, Palaeoclimatology, Palaeoecology 266:6982.

Eiselt, B. Sunday

2012 Becoming White Clay: A History and Archaeology of Jicarilla Apache Enclavement. University of Utah Press, Salt Lake City.

Ewers, John C.

1955 The Horse in Blackfeet Culture with Comparative Material from Other Western Tribes. Smithsonian Institution Bureau of American Ethnology Bulletin 159. U.S. Government Printing Office, Washington, DC.

Fewkes, Jesse Walter

1909 Antiquities of the Mesa Verde National Park SpruceTree House. Smithsonian Institution Bureau of American Ethnology Bulletin 41. U.S. Government Printing Office, Washington, DC.

Frison, George

1968 Daugherty Cave, Wyoming. Plains Anthropologist 13:253-295

Garling, Mary E.

1964 Ross Rock Shelter (48NA331). Wyoming Archaeologist 7(4):12-35.

Gilmore, Kevin P.

2005 These Boots Were Made for Walking: Moccasin Production, Gender, and the Late Prehistoric Hideworking Sequence on the High Plains of Colorado. In Gender and Hide Production, edited by Lisa Frink and Kathryn Weedman, pp. 13-36. AltaMira Press, Walnut Creek, California.

Gilmore, Kevin P., Derek Hamilton, and John W. Ives

2017 Promontory Culture in Eastern Colorado: Franktown Cave and Early Proto-Apachean Migration. Paper presented at the 82nd Annual Meeting of the Society for American Archaeology, Vancouver, British Columbia.

Gilmore, Kevin P., Jon Hedlund, and Bonnie Clark

2017 Through a Glass, Darkly: Shedding Light on Late Prehistoric Obsidian Conveyance and Ethnogenesis on the Plains. Paper presented at the Annual Meeting of the Colorado Council of Professional Archaeologists, Grand Junction, Colorado.

Gilmore, Kevin P., John W. Ives, and Derek Hamilton

2021 The Promontory Culture in Eastern Colorado: Franktown Cave and Early Proto-Apachean Migration. In New Perspectives on Apachean Origins, edited by 
John W. Ives and Joel C. Janetski. University of Utah Press, Salt Lake City, in press.

Gogan, Peter J. P., Nicholas C. Larter, James H. Shaw, John E. Gross, C. Cormack Gates, and Joe Truett

2010 General Biology, Ecology and Demographics. In American Bison: Status Survey and Conservation Guidelines, edited by C. Cormack Gates, Curtis H. Freese, Peter J. P. Gogan, and Mandy Kotzman, pp. 39-52. IUCN, Gland, Switzerland.

Graven, Heather, Colin E. Allison, David M. Etheridge, Samuel Hammer, Ralph F. Keeling, Ingeborg Levin, Harro A. J. Meijer, Mauro Rubino, Pieter P. Tans, Cathy M. Trudinger, Bruce H. Vaughn, and James W. C. White

2017 Compiled Records of Carbon Isotopes in Atmospheric $\mathrm{CO}_{2}$ for Historical Simulations in CMIP6. Geoscientific Model Development 10:4405-4417. DOI:10. 5194/gmd-10-4405-201.

Hallson, Jennifer

2017 A Quantitative Analysis of Promontory Cave 1: An Archaeological Study on Population Size, Occupation Span, Artifact Use-Life, and Accumulation. Master's thesis, Department of Anthropology, University of Alberta, Edmonton, Canada.

Hatt, Gudmund

1916 Moccasins and Their Relation to Arctic Footwear. Memoirs of the American Anthropological Association 3(3):149-250.

Hedges, Robert E. M., John G. Clement, C. David, L. Thomas, and Tamsin C. O'Connell

2007 Collagen Turnover in the Adult Femoral Mid-Shaft: Modeled from Anthropogenic Radiocarbon Tracer Measurements. American Journal of Physical Anthropology 133:808-816.

Hoard, Robert J., C. Tod Bevitt, and Janice McLean

2008 Source Determination of Obsidian from Kansas Archaeological Sites Using Compositional Analysis. Transactions of the Kansas Academy of Science 111:219-229.

Hobson, Keith A., and Robert G. Clark

1992 Assessing Avian Diets Using Stable Isotopes I: Turnover of ${ }^{13} \mathrm{C}$ in Tissues. Condor 94:181-188.

Hobson, Keith A., Don M. Schell, Deane Renouf, and Elizabeth Noseworthy

1996 Stable Carbon and Nitrogen Isotopic Fractionation between Diet and Tissues of Captive Seals: Implications for Dietary Reconstructions Involving Marine Mammals. Canadian Journal of Fisheries and Aquatic Sciences 53:528-533.

Hobson, Keith A., and Leonard I. Wassenaar

2019 Tracking Animal Migration with Stable Isotopes. Academic Press, London.

Hodell, David A., Rhonda L. Quinn, Mark Brenner, and George Kamenov

2004 Spatial Variation of Strontium Isotopes $\left({ }^{87} \mathrm{Sr} /{ }^{86} \mathrm{Sr}\right)$ in the Maya Region: A Tool for Tracking Ancient Human Migration. Journal of Archaeological Science 31:585601.

Hoppe, Kathryn A., Adina Paytan, and Page Chamberlain

2006 Reconstructing Grassland Vegetation and Paleotemperatures Using Carbon Isotope Ratios of Bison Tooth Enamel. Geology 34:649-652.

Hughes, Richard E.

2011 Energy Dispersive X-Ray Fluorescence Analysis of Obsidian Artifacts from Promontory Caves 1 and 2 (42BO1 and 42BO2), Great Salt Lake, Utah. Letter
Report 2011-57. Geochemical Research Laboratory, Portola Valley, California.

2012 Energy Dispersive X-Ray Fluorescence Analysis of Obsidian Artifacts from Promontory Cave 1 (42BO1), Great Salt Lake, Utah. Letter Report 2012-21. Geochemical Research Laboratory, Portola Valley, California.

Hughes, Richard E., Phil R. Geib, and Courtney L. C. Ziska 2019 Investigating Dismal River Obsidian Use in Central Nebraska. Plains Anthropologist 64:257-274.

Ives, John W.

2014 Resolving the Promontory Culture Enigma. In Archaeology in the Great Basin and Southwest: Papers in Honor of Don D. Fowler, edited by Nancy J. Parezo and Joel C. Janetski, pp. 149-162. University of Utah Press, Salt Lake City.

2020 The View from Promontory Point. In Spirit Lands of the Eagle and Bear: Numic Archaeology and Ethnohistory in the American West, edited by Robert H. Brunswig, pp. 90-117. University Press of Colorado, Boulder.

Ives, John W., Duane G. Froese, Joel C Janetski, Fiona Brock, and Christopher Bronk Ramsey

2014 A High Resolution Chronology for Steward's Promontory Culture Collections, Promontory Point, Utah. American Antiquity 79:616-637.

Ives, John W., Lindsay Johansson, R. W. Graham, and Courtney D. Lakevold

2014 Preliminary Observations on Bison Hunting and the Promontory Caves. Paper presented at the 34th Great Basin Anthropological Conference, Boise, Idaho.

Janetski, Joel C., and Grant C. Smith (editors)

2007 Hunter-Gatherer Archaeology in Utah Valley. Occasional Paper 12. Museum of Peoples and Cultures, Provo, Utah.

Johansson, Lindsay

2013 Promontory Culture: The Faunal Evidence. Master's thesis, Department of Anthropology, Brigham Young University, Provo, Utah.

Kohler, Timothy A., Scott G. Ortman, Katie E. Grundtisch, Carly M. Fitzpatrick, and Sarah M. Cole

2014 The Better Angels of Their Nature: Declining Violence through Time among Prehispanic Farmers of the Pueblo Southwest. American Antiquity 79:444-464.

Kohn, Matthew J.

2010 Carbon Isotope Compositions of Terrestrial $\mathrm{C}_{3}$ Plants as Indicators of (Paleo)ecology and (Paleo)climate. PNAS 107:19691-19695.

Kristensen, Todd J., Thomas D. Andrews, Glen MacKay, Ruth Gotthardt, Sean C. Lynch, M. John, M. Duke, Andrew J. Locock, and John W. Ives

2019 Identifying and Sourcing Pyrometamorphic Artifacts: Clinker in Subarctic North America and the Hunter-Gatherer Response to a Late Holocene Volcanic Eruption. Journal of Archaeological Science: Reports 23:773-790

Kristensen, Todd J., Alwynne B. Beaudoin, and John W. Ives

2020 Environmental and Hunter-Gatherer Responses to the White River Ash East Volcanic Eruption in the Late Holocene Canadian Subarctic. Arctic 73:153-186.

Kristensen, Todd J., P. Gregory Hare, Ruth M. Gotthardt, Norman A. Easton, John W. Ives, Robert J. Speakman, and Jeffrey T. Rasic

2019 The Movement of Obsidian in Subarctic Canada: Holocene Social Relationships and Human Responses to a Large-Scale Volcanic Eruption. Journal of Anthropological Archaeology 56:1-18. 
Lakevold, Courtney D.

2017 Space and Social Structure in the AD 13th Century Occupation of Promontory Cave 1, Utah. Master's thesis, Department of Anthropology, University of Alberta, Edmonton, Canada.

Lycett, Stephen J.

2015 Differing Patterns of Material Culture Intergroup Variation on the High Plains: Quantitative Analyses of Parfleche Characteristics vs. Moccasin Decoration. American Antiquity 80:714-731.

Martin, Jeff M., Rachel A. Martin, and Jim I. Mead 2017 Late Pleistocene and Holocene Bison of the Colorado Plateau. Southwestern Naturalist 62:14-28.

Matsubayashi, Jun, and Ichiro Tayasu

2019 Collagen Turnover and Isotopic Records in Cortical Bone. Journal of Archaeological Science 106:37-44.

Metcalfe, Jessica Z., and Jim I. Mead

2019 Do Uncharred Plants Preserve Original Carbon and Nitrogen Isotope Compositions? Journal of Archaeological Method and Theory 26:844-872.

Mooney, James

1898 Calendar History of the Kiowa Indians. Accompanying Paper in Seventeenth Annual Report of the Bureau of American Ethnology to the Secretary of the Smithsonian Institution, 1895-96, Part I, by J. W. Powell, pp. 129 144. U.S. Government Printing Office, Washington, DC.

Morden, Clifford, Jack D. Brotherson, and Bruce N. Smith

1986 Ecological Differences of $\mathrm{C}_{3}$ and $\mathrm{C}_{4}$ Plant Species from Central Utah in Habitats and Mineral Composition. Great Basin Naturalist 46:140-147.

Moulton, Gary E. (editor)

1987 The Definitive Journals of Lewis and Clark. University of Nebraska Press, Center for Great Plains Research, Lincoln.

O'Leary, Marion $\mathrm{H}$.

1988 Carbon Isotopes in Photosynthesis. Bioscience 38:328-336.

Ortman, Scott G., and Lynda D. McNeil

2017 The Kiowa Odyssey: Evidence of Historical Relationships among Pueblo, Fremont, and Northwest Plains Peoples. Plains Anthropologist 63:152-174.

Osborne, Carolyn Miles

2004 The Wetherill Collections and Perishable Items from Mesa Verde. N.p., Los Alamitos, California.

Reilly, Aileen

2015 Women's Work, Tools, and Expertise: Hide Tanning and the Archaeological Record. Master's thesis, Department of Anthropology, University of Alberta, Edmonton, Alberta, Canada.

Reimer, Paula J., Edouard Bard, Alex Bayliss, J. Warren Beck, Paul G. Blackwell, Christopher Bronk Ramsey, Caitlin E. Buck, Hai Cheng, R. Lawrence Edwards, Michael Friedrich, Pieter M. Grootes, Thomas P. Guilderson, Haflidi Haflidason, Irka Hajdas, Christine Hatte, Timothy J. Heaton, Dirk L. Hoffmann, Alan G. Hogg, Konrad A. Hughen, K. Felix Kaiser, Bernd Kromer, Sturt W. Manning, Mu Niu, Ron W. Reimer, David A. Richards, E. Marian Scott, John R. Southon, Richard A. Staff, Christian S. M. Turney, and Johannes van der Plicht

2013 IntCal13 and Marine13 Radiocarbon Age Calibration Curves 0-50,000 Years cal BP. Radiocarbon 55:1869-1887.

Robinson, Erick, Kyle Bocinsky, Darcy Bird, Jacob Freeman, and Robert L. Kelly
2021 Dendrochronological Dates Confirm a Late Prehistoric Population Decline in the American Southwest Derived from Radiocarbon Dates. Philosophical Transactions of the Royal Society B: Biological Sciences 376:20190718. DOI:10.1098/rstb.2019.0718.

Rutley, Bruce D., and Robert J. Hudson

2000 Seasonal Energetic Parameters of Free-Grazing Bison (Bison bison). Canadian Journal of Animal Science 80:663-671.

Sandquist, Darren R., and James R. Ehleringer

1995 Carbon Isotope Discrimination in the $\mathrm{C}_{4}$ Shrub Atriplex confertifolia along a Salinity Gradient. Great Basin Naturalist 55:135-141.

Schaefer, Allan L., Bruce A. Young, and Andrew M. Chimwano

1978 Ration Digestion and Retention Times of Digesta in Domestic Cattle (Bos taurus), American Bison (Bison bison), and Tibetan Yak (Bos grunniens). Canadian Journal of Zoology 56:2355-2358.

Schmitt, Jochen, Robert Schneider, Joachim Elsig, Daiana Leuenberger, Anna Lourantou, Jerome Chappellaz, Peter Koehler, Fortunat Joos, Thomas F. Stocker, Markus Leuenberger, and Hubertus Fischer

2012 Carbon Isotope Constraints on the Deglacial $\mathrm{CO}_{2}$ Rise from Ice Cores. Science 336:711-714.

Seymour, Deni J.

2010 Contextual Incongruities, Statistical Outliers, and Anomalies: Targeting Inconspicuous Occupational Events. American Antiquity 75:158-176.

2012 "Big Trips" and Historic Apache Movement and Interaction: Models for Early Athapaskan Migrations. In From the Land of Ever Winter to the American Southwest: Athapaskan Migrations, Mobility, and Ethnogenesis, edited by Deni J. Seymour, pp. 377-409. University of Utah Press, Salt Lake City.

Sponheimer, Matt, Todd Robinson, Linda Ayliffe, Ben Passey, Beverly Roeder, Lisa Shipley, Elvia Lopez, Thure Cerling, Denise Dearing, and Jim Ehleringer

2003 An Experimental Study of Carbon-Isotope Fractionation between Diet, Hair, and Feces of Mammalian Herbivores. Canadian Journal of Zoology 81:871876.

Sternberg, Leonel O., Michael J. DeNiro, and Hyrum B. Johnson

1984 Isotope Ratios of Cellulose from Plants Having Different Photosynthetic Pathways. Plant Physiology 74:557-561.

Steward, Julian Haynes

1937 Ancient Caves of the Great Salt Lake Region. Bureau of American Ethnology Bulletin 115. Smithsonian Institution, Washington, DC.

Szpak, Paul, Jessica Z. Metcalfe, and Rebecca A. Macdonald 2017 Best Practices for Calibrating and Reporting Stable Isotope Measurements in Archaeology. Journal of Archaeological Science: Reports 13:609-616.

Thomas, David Hurst

2018 A Shoshonean Prayerstone Hypothesis: Ritual Cartographies of Great Basin Incised Stone. American Antiquity 84:1-25.

Tieszen, Larry

1994 Stable Isotopes on the Plains: Vegetation Analysis and Diet Determinations. In Skeletal Biology in the Great Plains: Migration, Warfare, Health, and Subsistence, edited by Douglas W. Owsley and Richard L. Jantz, pp. 261-282. Smithsonian Institution, Washington, DC. 
Ugan, Andrew, and Joan Coltrain

2012 Stable Isotopes, Diet, and Taphonomy: A Look at Using Isotope-Based Dietary Reconstructions to Infer Differential Survivorship in Zooarchaeological Assemblages. Journal of Archaeological Science 39:14011411.

van Klinken, Gert J.

1999 Bone Collagen Quality Indicators for Paleodietary and Radiocarbon Measurements. Journal of Archaeological Science 26:687-695.

Webber, Alika Podolinsky

1989 North American Indian and Eskimo Footwear: A Typology and Glossary. Bata Shoe Museum, University of Toronto Press, Toronto.

Wilson, Gilbert Livingstone

1924 The Horse and the Dog in Hidatsa Culture. Anthropological Papers of the American Museum of Natural History Vol. 15, Pt. 2. American Museum Press, New York.

Wood, Rachel E., Christopher Bronk Ramsey, and Thomas F. G. Higham

2016 Refining Background Corrections for Radiocarbon Dating of Bone Collagen at ORAU. Radiocarbon 52:600-611.
Yanicki, Gabriel M.

2019 Promontory-Fremont Contact and Ethnogenesis in the Post-Formative Eastern Great Basin. PhD dissertation, Department of Anthropology, University of Alberta, Edmonton, Alberta, Canada.

Yanicki, Gabriel M., and John W. Ives

2017 Mobility, Exchange, and the Fluency of Games: Promontory in a Broader Sociodemographic Setting. In Prehistoric Games of North American Indians: Subartic to Mesoamerica, edited by Barbara Voorhies, pp. 139-162. University of Utah Press, Salt Lake City.

Zazula, Grant D., Ross D. E. MacPhee, Jessica Z. Metcalfe, Alberto V. Reyes, Fiona Brock, Patrick S. Druckenmiller, Pamela Groves, C. Richard Harington, Gregory W. L. Hodgins, Michael L. Kunz, Fred J. Longstaffe, Daniel H. Mann, H. Gregory McDonald, Shweta NalawadeChavan, and John R. Southon

2014 American Mastodon Extirpation in the Arctic and Subarctic Predates Human Colonization and Terminal Pleistocene Climate Change. PNAS 111:18460-18465.

Submitted January 23, 2020; Revised August 29, 2020;

Reviews completed October 5, 2020; Accepted October 5, 2020 